\title{
Demersal assemblages of the continental shelf and slope edge between the Gulf of Tehuantepec (Mexico) and the Gulf of Papagayo (Costa Rica)
}

\author{
G. Bianchi \\ Institute of Marine Research, Division for International Development Programmes, PO Box 1870, Nordnes, N-5024 Bergen, \\ Norway
}

\begin{abstract}
The structure of demersal assemblages (fish, crustaceans and cephalopods) of the continental shelf and upper slope between the Gulf of Tehuantepec and the Gulf of Papagayo was studied from data obtained in the course of surveys carried out by the RV 'Dr. F. Nansen' in 1987, by means of an ordination technique, Detrended Correspondence Analysis (DCA) implemented by the program DECORANA, and a classification technique, Two-Way Indicator Species Analysis (TWIA) implemented by the program TWINSPAN. Three major groups of species were identified: those distributed above the thermocline, those within the range of the thermocline and a third group below the thermocline, where oxygen content is extremely low. Highest biomass densities were found below the thermocline, consisting mainly of the galatheid crustacean Pleuroncodes monodon (H. Milne Edwards, 1837). Correlation of DCA Axis 1 with depth, temperature, salinity and oxygen showed that depth is the main gradient along which faunal changes occur.
\end{abstract}

\section{INTRODUCTION}

Since 1975 the Norwegian RV 'Dr. F. Nansen' has carried out acoustic and trawl surveys of the continental shelves and upper slopes of many tropical countries. The present study is the first of a series based on material from these surveys, investigating the structure of demersal assemblages in relation to principal environmental variables and geographical location. The taxa included in the analysis are bony and cartilaginous fishes, stomatopods, decapod crustaceans and cephalopods.

As pointed out by Caddy \& Sharp (1986), this type of study is a necessary step toward understanding of multispecies stocks. Such work can then be extended to 'descriptive community dynamics' (McManus 1985) in order to find general patterns of which species compositions can be expected under given environmental conditions and fishing effort. Comparison of assemblages from similar ecosystems in different areas might also reveal general trends in the community dynamics of tropical shelves.

In addition, this work could be useful in fisheries management. For example, species composition of trawl catches from a given study area may be roughly anticipated from assemblage maps derived from the analysis, especially for those areas most recently investigated.

Studies of tropical fish community structure by means of multivariate analysis (excluding coral reef areas and lagoon systems) have been carried out in the Gulf of Guinea (Fager \& Longhurst 1968), Namibia (Lleonart \& Roel 1984), upwelling areas of West Africa (Roel et al. 1985), the Gulf of Nicoya, Costa Rica (Bartels et al. 1983), the Samar Sea, Philippines (McManus 1985), Malaysia (Chan \& Liew 1986), northern Australia (Rainer \& Munro 1982 and Rainer 1984) and northwestern Australia (Sainsbury 1987).

To the author's knowledge, no other studies of shelf assemblages have covered the area considered in the present work, i.e. the shelf between the Gulf of Tehuantepec and the Gulf of Papagayo. Bartels et al. (1983) described the occurrence, distribution, abundance and diversity of fish assemblages in the Gulf of Nicoya, Costa Rica. Studies on fish community structure in coastal lagoon systems on the Pacific coast of Mexico were carried out by Warburton (1978), YanezArancibia (1978 a, b) and Chavez (1979). 

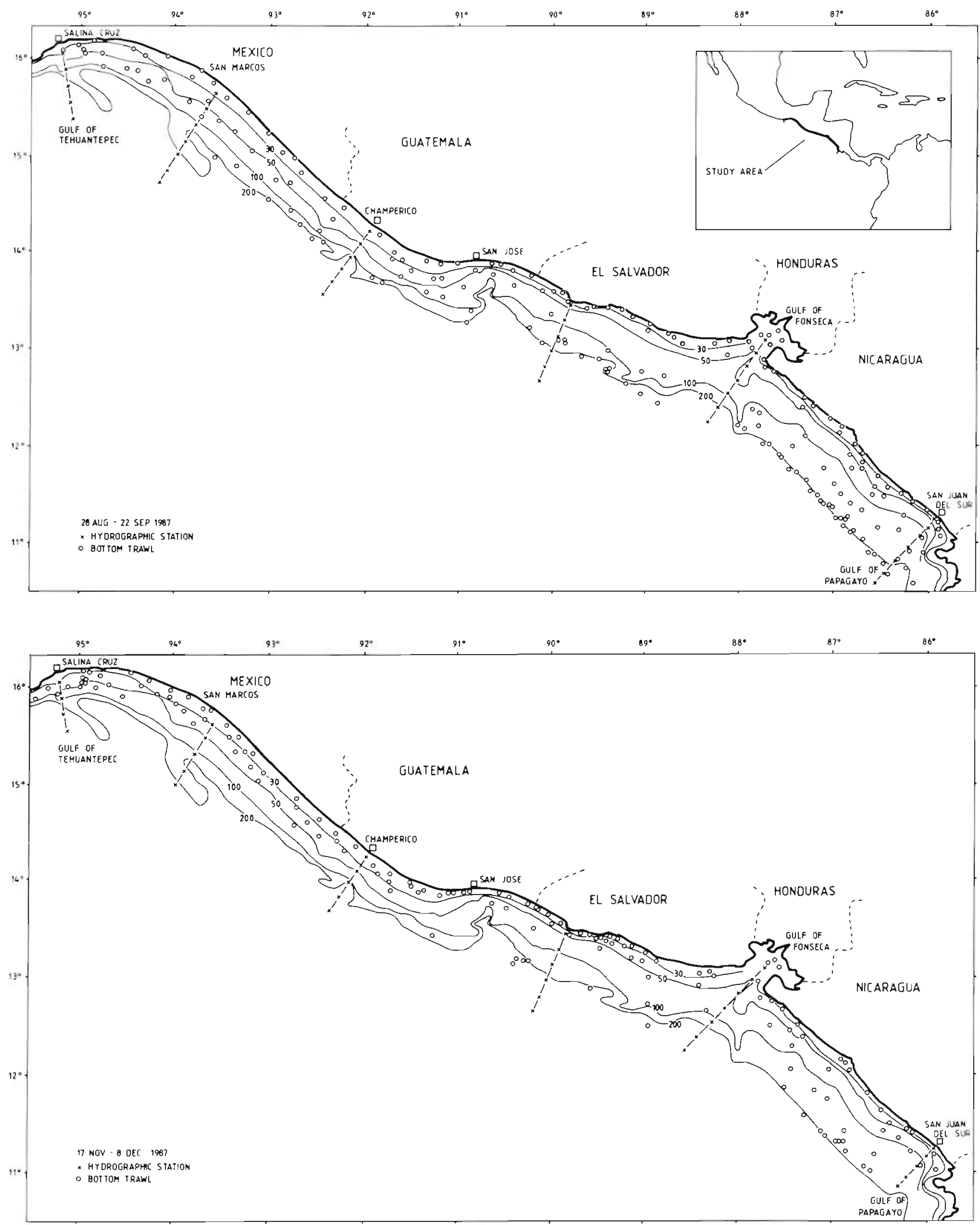

Fig. 1. Position of trawl hauls and hydrographic stations in 1987. Upper: August-September survey; lower: NovemberDecember survey. Depth gradients in metres 


\section{STUDY AREA}

The study area (Fig. 1) included a coastline of about 685 nautical miles, from $95^{\circ} 30^{\prime} \mathrm{W}$ (Mexico) to $85^{\circ} 50^{\prime} \mathrm{W}$ (northern Costa Rica), and an area of about 28300 square nautical miles (from about 10 to $500 \mathrm{~m}$ depth), of which 23000 represent the shelf area to 200 $m$ depth and about 5300 represent the upper slope (Strømme \& Sætersdal 1988). Off Nicaragua and El Salvador the shelf is wide; it narrows off Guatemala and widens again off southern Mexico. The shelf bottom is muddy throughout, but sand and shells are dominant off southern Mexico (Anonymous 1977). The slope to $500 \mathrm{~m}$ depth is quite steep off Nicaragua and northwards to Guatemala. Off Mexico it is much wider, with steep and rough bottoms especially in the northwest.

Water masses of the eastern tropical Pacific are comprehensively described by Wyrtki (1967). Hydrographic conditions on the shelf area during the survey period are described in the survey report (Stromme and Sæetersdal 1988). The biological oceanography of the eastern tropical Pacific has been reviewed by Blackburn (1966).

The surface offshore circulation of this area is characterized by the Costa Rica Current, i.e. the north branch of the Equatorial Counter Current which splits when approaching Costa Rica. Strongest from June to December, this current flows parallel to the coast and around the Costa Rica Dome and turns westward to feed the North Equatorial Current.
An oxygen-minimum layer more than $1200 \mathrm{~m}$ thick characterizes the intermediate water masses off Mexico to Costa Rica; its upper boundary is described as being shallower than $50 \mathrm{~m}$ in the coastal and offshore areas from about $9^{\circ} \mathrm{N}$ (Costa Rica) to $16^{\circ} \mathrm{N}$ (southern Mexico). This oxygen-minimum layer is a consequence of sluggish water movement in these areas where circulation of the subtropical anticyclones does not penetrate (Wyrtki 1967). In the course of our survey, oxygen levels of $1 \mathrm{ml} \mathrm{l}^{-1}$ were observed on the shelf bottom between 50 and $150 \mathrm{~m}$ depth, varying with geographical location and season and with a tendency to occur in shallower waters towards the north. In September this level was found between $75 \mathrm{~m}$ (Salina Cruz, Mexico) and $125 \mathrm{~m}$ (San Juan del Sur, Nicaragua), while in November/December it ascended and was located at about 50 and $75 \mathrm{~m}$ respectively. Below the $1 \mathrm{ml} \mathrm{l}^{-1}$ isoline, oxygen content decreased and levels of $0.5 \mathrm{ml} \mathrm{l}^{-1}$ were found at the edge of the continental shelf or upper slope throughout the year (Strømme \& Sætersdal 1988). Fig. 2 shows oxygen profiles at selected stations, for both warm and cold seasons.

The thermocline is shallow, located between about 35 and $100 \mathrm{~m}$ and present all year round. It appears to be slightly shallower during the cold (upwelling) season (Fig. 3). Coastal upwelling occurs as a consequence of the strong northeast trade winds, from November to April, through the mountain gaps of southern Mexico and southern Nicaragua/northern Costa Rica. Upwelling in the Gulf of Tehuantepec is

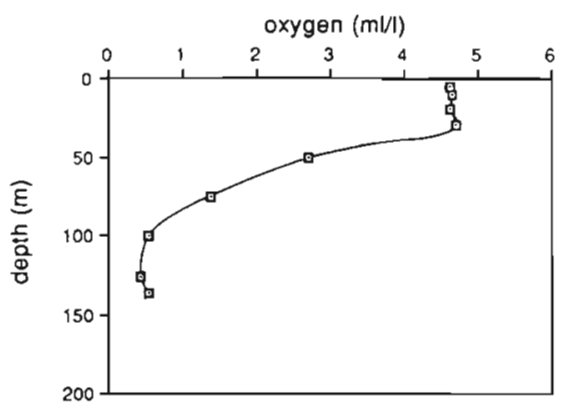

a) Salina Cruz (Aug.-Sept. survey)

c) Salina Cruz (Nov.-Dec. Survey)

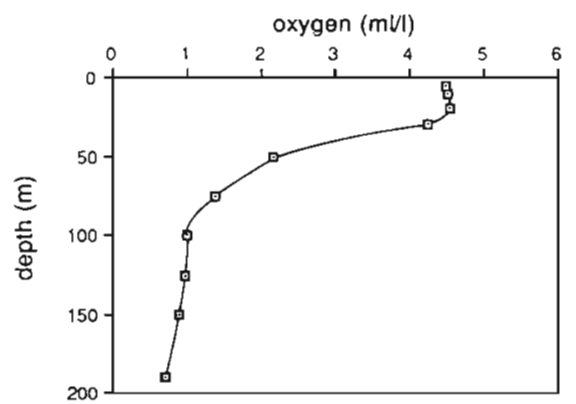

b) San Juan del Sur (Aug. -Sept. survey)

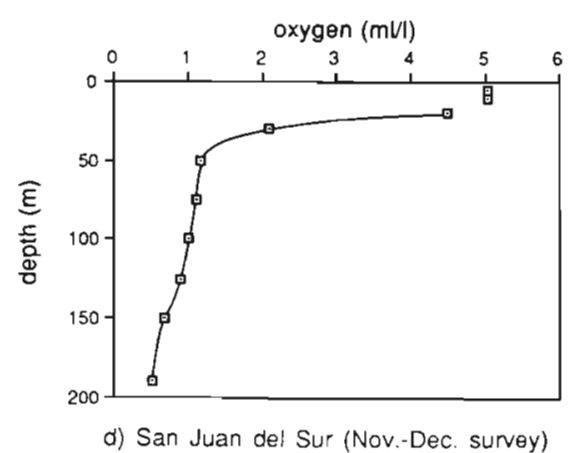

d) San Juan del Sur (Nov-Dec. survey)
Fig. 2. Oxygen concentrations $\left(\mathrm{ml} \mathrm{l}^{-1}\right)$ in relation to depth at selected stations in 1987

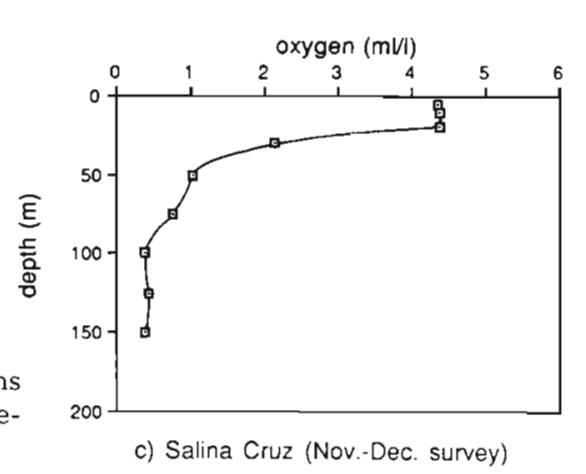




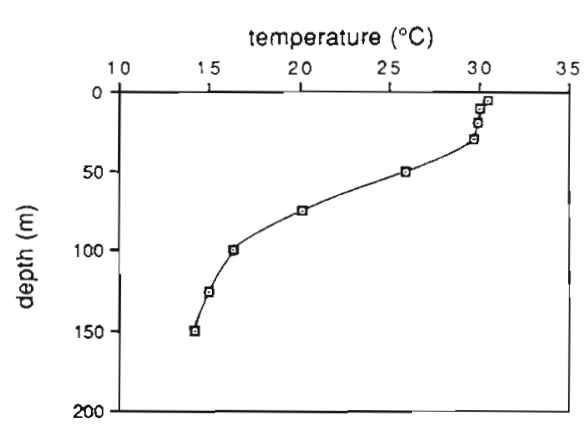

a) Salina Cruz (Aug.-Sept. survey)

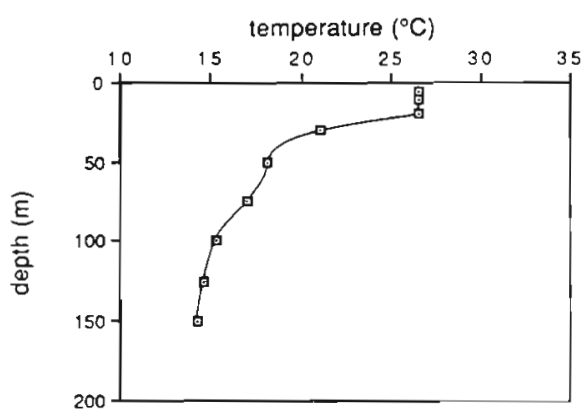

c) Salina Cruz (Nov.-Dec. Survey)

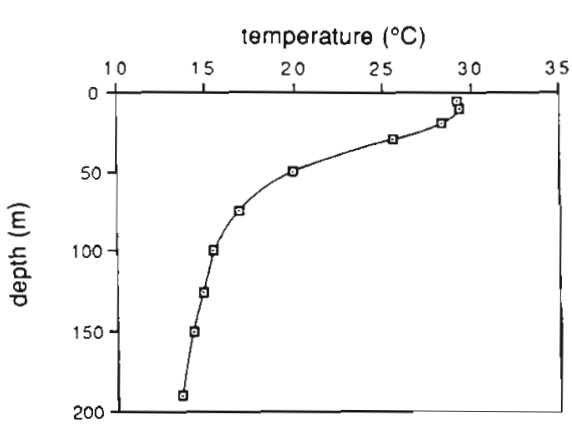

b) San Juan del Sur (Aug.-Sept. Survey)

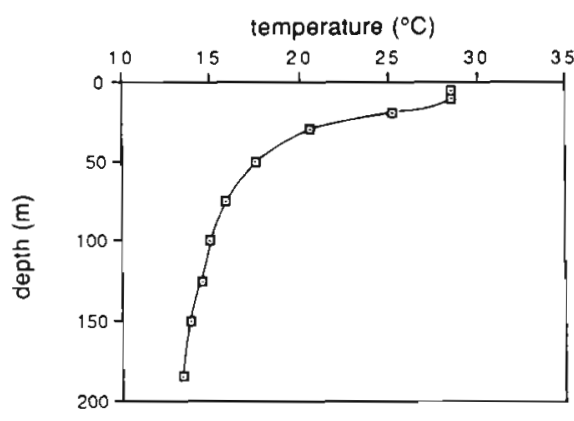

d) San Juan del Sur (Nov-Dec. survey)
Fig. 3. Temperature in relation to depth at selected stations in 1987 described by Roden (1961). Upwelling in the Gulf of Papagayo is also a well-known event. Temperatures below $20^{\circ} \mathrm{C}$ have been observed in the surface waters near San Juan del Sur (Nicaragua), lasting about 3 mo (January to March; Glynn et al. 1983). Hydrographic observations made with the RV 'Dr. F. Nansen' showed a clear upward trend in depth of the thermocline in the course of Survey 3 (in December 1987). Offshore upwelling has been described for the Costa Rica Dome by Wyrtki (1967), and the associated higher productivity by Blackburn (1966). More detailed studies on phytoplankton and copepod distribution in this area are presented by Sameoto (1986) and Subba-Rao \& Sameoto (1988); the latter compared vertical distribution $(0$ to $1000 \mathrm{~m})$ of phytoplankton inside and outside the Dome area and reported a much higher phytoplankton concentration inside the area, at all depths. Higher concentrations were also found in the aphotic zone originating from the overlying euphotic waters, and this probably plays an important role in the food web of the deep sea.

The shoal thermocline seems also to be a cause of high productivity. Brandhorst (1958) found that in areas of the eastern Pacific where the thermocline was close to the surface $(20$ to $50 \mathrm{~m})$, standing stocks of chlorophyll and zooplankton were higher than in areas with a deeper thermocline. This is probably due to enrichment from below by wind-mixing and to the significantly higher productivity in the well-lit waters below the mixed layer (Blackburn 1966).

\section{MATERIALS AND METHODS}

Trawl data. Material was collected on 2 cruises in 1987 (28 August to 21 September and 17 November to 7 December, respectively). A shrimp and fish trawl was used, with a headline of $31 \mathrm{~m}$, footrope of $47 \mathrm{~m}$, and estimated headline height and distance between wings during towing of 6 and 18-20 m respectively. Mesh size was $2 \mathrm{~cm}$, with double lining in the cod end. Each tow had a standard duration of $30 \mathrm{~min}$. Other details on the gear used may be found in Stromme \& Sæetersdal (1988). The bottom-trawl stations used in this analysis were randomly set along the cruise track, but in the November-December survey higher effort was concentrated in the inshore areas and at the edge of the continental shelf and upper slope off Nicaragua. The present analysis is based mainly on the August-September survey, with a higher and better-distributed effort, while the November-December survey was used for comparison. A total of 191 and 157 stations were sampled in the course of the 2 surveys respectively (Fig. 1a, b).

Each specimen caught was counted and weighed separately. In cases where identification was possible only to genus or family, provisional names were given and specimens were retained and later identified by experts on the various groups. For the present analysis congeneric species which were difficult to separate were pooled together.

A large collection of bony fishes and crustaceans was deposited at the Senckenberg Museum (Frankfurt, 
Germany). All station and species data were stored using the B-trieve file system (data available in ASCII format upon request to the author).

Hydrographic data. Samples for temperature, salinity and oxygen measurements were taken with Nansen bottles at standard depths, along fixed transects (Fig. $3 \mathrm{a}, \mathrm{b})$. Surface temperature ( $4 \mathrm{~m}$ depth) was continuously recorded by a thermograph. Details on oceanographic data can be found in Strømme \& Sætersdal (1988, Vol. 2: Data File)

Temperature, salinity and oxygen data were used in the present analysis to examine relationships of the different species assemblages to the physical environment. For the above variables, values were assigned to each trawl station from the nearest hydrographic station at a similar depth.

Data analysis. The primary objective was to identify major patterns of species associations based on the trawl data, to relate them to the more significant environmental factors and hence to explain the observed patterns. The method traditionally used in fish-community studies to identify groups of species/ samples has been cluster analysis, usually using an agglomerative clustering algorithm. This method produces a classification diagram (dendrogram) which also shows the hierarchical relationships between groups. Drawbacks of this method are the production of miscellaneous clusters from 'left-overs' or chaining, i.e. adding objects one-by-one to groups to which they do not really belong. Also, it is quite difficult to relate the sample dendrogram to the species dendrogram and understand which species group corresponds to a given sample cluster.

Two-Way Indicator Species Analysis - TWIA (Hill 1979), implemented by the computer program TWINSPAN - was considered well suited to the main objectives of this work. This method involves a primary ordination of the samples by correspondence analysis (see below) and divisions near the midpoint of each principal axis from each successive analysis, so that each division serves to contrast the most dissimilar object types. The method :. . constructs a classification of the samples, and then uses this classification to obtain a classification of the species according to their ecological preferences. The two classifications are then used together to obtain an ordered two-way table that expresses the species' synecological relations as succinctly as possible' (Hill 1979). In addition to a hierarchical classification of samples and species, TWIA produces a sorted community table in which stations and species are arranged along the major gradients within the data. Importance values are not used directly but are converted to a scale based on lower class limits (set at 0,10,100,1000 and $10000 \mathrm{~kg}$ in this study, according to catch size by species, which varied from 0 to ca $20000 \mathrm{~kg}$ ).
Detrended Correspondence Analysis (DCA; Hill \& Gauch 1980), implemented by the computer program DECORANA, was used as a complementary ordination method. This method is particularly useful in ecological studies as it does not assume linear relationships between species abundances and environmental variables. It implicitly assumes a simple unimodal speciesresponse model (ter Braak \& Prentice 1988). DCA is a heuristic modification of Correspondence Analysis (CA), developed to eliminate the 'arch effect' and the distortion of relative distances in the ordination which are characteristic of $\mathrm{CA}$. The particular version used in this study (from the program package CANOCO; ter Braak 1987) provides the option of detrending by second-order polynomials (ter Braak \& Prentice 1988) instead of by segments as in the original version of the program DECORANA (Hill \& Gauch 1980). Detrending by second-order polynomials seems to avoid the inconvenience of destruction of ecologically meaningful information which might occur when detrending by segments (Jongman et al. 1987) and was thus used in the present study.

The above methods are both based on correspondence analysis, which makes it possible to compare their results directly, i.e. the classification from TWINSPAN and the ordination along the first axis of DCA. Comparison is useful, as outliers can affect site classification and can be identified through the ordination results.

The relationship between station groups and environmental variables was analyzed using the DCA application in the program package CANOCO, which also provides the option of correlating the ordination axes with environmental variables (depth, temperature, salinity, and oxygen). This option also produces the mean and SD of the environmental variables for each group.

A table of 'pseudo- $F^{\prime}$ values (ratios of among-group to within-group variances) was constructed to evaluate the degree of conformity of a given species to a site group obtained from the above methods. A formal $F$ test cannot be performed in this case because it would be based on the same data previously used to establish the groups (Green \& Vascotto 1978).

In this study biomass (wet weight) was used as a measure of abundance. Biomass is of more relevance to fisheries management and seems ecologically appropriate.

Each weight $(x)$ was converted to $\ln (x+1)$ before DCA and the 'pseudo- $F$ ' test were performed. This transformation minimizes the dominant effect of anomalous catches. The addition of 1 unit is necessary to avoid problems derived by the presence of values = 0 or values $<1$. Trials on a small sample of stations showed that this transformation did not affect the results. No transformation is necessary in the case of 
TWIA, where abundances are converted to numbers corresponding to different abundance classes (socalled pseudospecies).

Demersal biomass densities (weight per unit area) were calculated using the 'swept area' method, by depth stratum:

$$
D_{j}=C_{j} / q a_{j}
$$

where $D_{j}=$ density in Stratum $j$ (tonnes per nautical mile), $C_{j}=$ catch taken in hauls in Stratum $j$ (tonnes), $a_{j}=$ area of the bottom 'swept' by the trawl hauls in Stratum $j$ (square nautical miles), $q=$ catchability coefficient $(=1$, i.e. all fish in the path of the trawl were caught).

Sampling errors and limitations. The research vessel could only operate in waters deeper than 10 to $15 \mathrm{~m}$. Therefore, shallow-water communities were insufficiently sampled.

Bottom trawls are both species- and size-selective, and it was impossible to adjust for this type of selectivity without knowing the behaviour of most species or the real age/size structure of populations. Also, in the case of long tows, the trawl might have artificially blended different assemblages occurring within the path of the trawl.

Species identification often poses serious problems in tropical areas. Unfortunately, no guides such as the F.A.O. Species Identification Sheets for fishery purposes were available for the eastern central Pacific. Although taxonomic work was carried out with the participation of well-trained taxonomists, errors in identification may have occurred because of the participation of less-trained personnel.

Effort (i.e. number of stations) was not uniformly distributed in space or time, and this might have led to biased results. In fact many species, both demersal and pelagic, show important day/night variations in behaviour pattern, but a comparison between day and night catches was not possible because most of the deep-water stations were sampled at night, while stations in shallower water were sampled during the day. This choice was deliberate, based on patterns observed with the echo-integration system indicating that bottom fish tend to be closer to the bottom during daytime while a large number of species move to upper water layers at night. This phenomenon appears to be less pronounced in the deeper part of the shelf and upper slope.

Many typically pelagic species are often caught in bottom trawls. In shallow waters (10 to $20 \mathrm{~m}$ ), it is quite difficult to differentiate between these 2 groups: small pelagic fish of this zone are also found quite close to the bottom, as some of them feed on bottom detritus and are preyed upon by both demersal and pelagic predators. It seems that in these very shallow waters, demersal and pelagic groups have a much closer relationship than in more offshore waters. For this reason, although this analysis is mainly aimed at demersal communities, pelagic species were included in the analysis whenever they occurred in the bottom trawl. In the deeper part of the shelf and upper slope, some pelagic and mesopelagic species which perform diumal vertical migrations were caught in bottom trawls during daytime. Even when this occurred the species were included in the analysis, although the results were interpreted in the light of this information.

\section{RESULTS}

A total of 230 species comprising 16004372 specimens (203 $155 \mathrm{~kg}$ ) were sampled. Table 1 gives a list of the most important species collected and used in the analysis.

Appendix 1 shows the 2-way classification of species and stations obtained with TWINSPAN, while Fig. 4 shows the TWIA dendrogram for station groups. The first dichotomy separates all the stations shallower than $100 \mathrm{~m}$ (Groups 1 to 6) from those on the deeper part of the shelf and upper slope (Groups 7 and 8), where oxygen levels are well below $1 \mathrm{ml} \mathrm{l}^{-1}$. At the second division level Group 6 (the intermediate shelf-dwellers, at depths between 50 and $100 \mathrm{~m}$ ) is separated from Groups 1 to 5, and Group 7 from Group 8. Further divisions of the deeper stations were not considered as they seemed to be mainly due to day/night variations in the catches. At the third division level Group 1 (stations at about 30 to $40 \mathrm{~m}$ depth, on sandy/shell bottoms off Guatemala and Mexico) is separated from the very shallow stations (Groups 2 to 4 ) and from the corresponding depth range in the southern part, off Nicaragua and El Salvador (Group 5). Finally, Group 4, including the Gulf of Fonseca and the adjacent shallow waters, is separated from the remaining shallow coastal waters (Groups 2 and 3 ) in the fifth division.

Fig. 5 shows the ordination of the stations from the August-September survey on DCA Axes 1 and 2. The eigenvalues of the first 4 axes were $0.92,0.43,0.28$ and 0.24 respectively. This shows that the gradient represented by the first axis is by far the most important. The 2 largest discontinuities along the first axis $(0.42$ and $0.63 \mathrm{SD}$, respectively) produce 3 groups: a first group including stations usually shallower than $50 \mathrm{~m}$, a second group of stations between 50 and $100 \mathrm{~m}$, and a third group in which most stations were deeper than $150 \mathrm{~m}$.

Results from the correlation of DCA Axes 1 and 2 with the environmental variables are presented in Table 2. The first axis was highly correlated with depth, temperature and oxygen, while there was no significant correlation of these variables with Axis 2 . 
Table 1. Main species collected in 1987 between the Gulf of Tehuantepec (Mexico) and the Gulf of Papagayo (Costa Rica), by major taxonomic groups and families

\begin{tabular}{|c|c|c|}
\hline Cephalopods & Ariidae & Haemulidae \\
\hline Loliginidae & Arius spp. & Conodon macrops Hildebrand \\
\hline Loliolopsis diomedeae (Hoyle) & Bagre panamensis (Gill) & Orthopristis chalceus (Günther) \\
\hline Lolliguncula panamensis Berry & Galeichthys peruvianus Lütken & Pomadasys axillaris (Steindachner) \\
\hline Stomatopods & Argentinidae & Pornadasys leuciscus (Günther) \\
\hline Squillidae & Argentina aliceae Cohen & $\begin{array}{l}\text { Pomadasys panamensis (Stein- } \\
\text { dachner) }\end{array}$ \\
\hline Squilla biformis Bigelow & Synodontidae & Xenichthys xanti (Gill) \\
\hline Squilla panamensis Bigelow & $\begin{array}{l}\text { Synodus evermanni Jordan \& } \\
\text { Bollman }\end{array}$ & Sciaenidae \\
\hline Decapod crustaceans & Synodus scituliceps Jordan \& Gilbert & Bairdiella spp. \\
\hline $\begin{array}{l}\text { Solenoceridae } \\
\text { Solenocera agassizii Faxon }\end{array}$ & Myctophidae & $\begin{array}{l}\text { Cynoscion phoxocephalus Jordan \& } \\
\text { Gilbert }\end{array}$ \\
\hline Penaeidae & Moridae & $\begin{array}{l}\text { Cynoscion reticulatus (Günther) } \\
\text { Cynoscion stolzmanni (Steindachner) }\end{array}$ \\
\hline Penaeus brevirostris Kingsley & Merlucciidae & Isopisthus altipinnis (Steindachner) \\
\hline Penaeus californiensis Holmes & Merluccius angustimanus Garman & Larimus acclivis Jordan \& Bristol \\
\hline $\begin{array}{l}\text { Penaeus vannamei Boone } \\
\text { Xiphopenaeus riveti Bouvier }\end{array}$ & $\begin{array}{l}\text { Ophidiidae } \\
\text { Lepophidium pardale (Gilbert) }\end{array}$ & $\begin{array}{l}\text { Larimus effulgens Gilbert } \\
\text { Larimus gulosus Hildebrand }\end{array}$ \\
\hline $\begin{array}{l}\text { Pandalidae } \\
\text { Heterocarpus sp. }\end{array}$ & Batrachoididae & $\begin{array}{l}\text { Micropogonias altipinnis (Günther) } \\
\text { Stellifer spp. }\end{array}$ \\
\hline $\begin{array}{l}\text { Galatheidae } \\
\text { Pleuroncodes monodon (H. Milne } \\
\text { Edwards) }\end{array}$ & $\begin{array}{l}\text { Lophiidae } \\
\text { Lophiodes caulinaris (Garman) }\end{array}$ & $\begin{array}{l}\text { Mullidae } \\
\text { Pseudupeneus grandisquamis (Gill) } \\
\text { Ephippididae }\end{array}$ \\
\hline $\begin{array}{l}\text { Calappidae } \\
\text { Mursia gaudichaudii (H. Milne } \\
\text { Edwards) }\end{array}$ & $\begin{array}{l}\text { Ogcocephalidae } \\
\text { Zalieutes elater (Jordan \& Gilbert) } \\
\text { Scorpaenidae }\end{array}$ & $\begin{array}{l}\text { Chaetodipterus zonatus (Girard) } \\
\text { Parapsettus panamensis (Stein- } \\
\text { dachner) }\end{array}$ \\
\hline $\begin{array}{l}\text { Portunidae } \\
\text { Portunus acuminatus (Stimpson) } \\
\text { Portunus asper (A. Milne Edwards) }\end{array}$ & $\begin{array}{l}\text { Pontinus sierra (Gilbert) } \\
\text { Scorpaena spp. }\end{array}$ & $\begin{array}{l}\text { Sphyraenidae } \\
\quad \text { Sphyraena ensis Jordan \& Gilbert }\end{array}$ \\
\hline $\begin{array}{l}\text { Sharks } \\
\text { Carcharhinidae } \\
\text { Carcharhinus porosus (Ranzani) }\end{array}$ & $\begin{array}{l}\text { Triglidae } \\
\text { Prionotus horrens Richardson } \\
\text { Prionotus quiescens Jordan \& } \\
\text { Bollman }\end{array}$ & $\begin{array}{l}\text { Polynemidae } \\
\text { Polydactylus approximans (Lay \& } \\
\text { Bennet) } \\
\text { Polydactylus opercularis (Gill) }\end{array}$ \\
\hline Nasolamia velox Gilbert & Serranidae & Gobiidae \\
\hline $\begin{array}{l}\text { Sphyrnidae } \\
\text { Sphyrna lewini (Cuvier, Griffith \& } \\
\text { Sinith) }\end{array}$ & $\begin{array}{l}\text { Diplectrum euryplectrum Jordan \& } \\
\text { Bollman } \\
\text { Diplectrum labarum Rosenblatt \& } \\
\text { Johnson }\end{array}$ & $\begin{array}{l}\text { Trichiuridae } \\
\text { Trichiurus nitens Garman } \\
\text { Scombridae }\end{array}$ \\
\hline Bony fishes & Diplectrum macropoma (Günther) & Scomberomorus sierra Jordan \& \\
\hline $\begin{array}{l}\text { Albulidae } \\
\text { Albula vulpes (Linnaeus) }\end{array}$ & $\begin{array}{l}\text { Hemanthias signifer (Garman) } \\
\text { Pronotogrammus eos Gilbert }\end{array}$ & $\begin{array}{l}\text { Starks } \\
\text { Stromateidae }\end{array}$ \\
\hline Muraenidae & Carangidae & Peprilus snyderi Gilbert \& Starks \\
\hline Ophichthidae & Caranx caballus Günther & Bothidae \\
\hline $\begin{array}{l}\text { Clupeidae } \\
\text { Neoopisthopterus tropicus } \\
\text { (Hildebrand) } \\
\text { Opisthonema libertate (Günther) }\end{array}$ & $\begin{array}{l}\text { Chloroscombrus orqueta Jordan \& } \\
\text { Gilbert } \\
\text { Carangoides ortrynter (Jordan \& } \\
\text { Gilbert) }\end{array}$ & $\begin{array}{l}\text { Citharichthys platophrys Gilbert } \\
\text { Cyclopsetta querna (Jordan \& } \\
\text { Bollman) } \\
\text { Monolene maculipinna Garman }\end{array}$ \\
\hline $\begin{array}{l}\text { Opisthopterus dovii (Günther) } \\
\text { Opisthopterus equitorialis }\end{array}$ & Hemicaranx spp. & Cynoglossidae \\
\hline $\begin{array}{l}\text { Opisthopterus equitorialis } \\
\text { (Hildebrand) }\end{array}$ & Oligoplites refulgens Gilbert \& Starks & Symphurus spp. \\
\hline $\begin{array}{l}\text { Pliosteostoma lutipinnis (Jordan \& } \\
\text { Gilbert) }\end{array}$ & $\begin{array}{l}\text { Selar crumenophthalmus (Bloch) } \\
\text { Selene peruvianus (Guichenot) }\end{array}$ & $\begin{array}{l}\text { Symphurus atramentatus Jordan \& } \\
\text { Bollman }\end{array}$ \\
\hline $\begin{array}{l}\text { Gilbert) } \\
\text { Engraulididae }\end{array}$ & Lutjanidae & Symphurus elongatus (Günther) \\
\hline $\begin{array}{l}\text { Engraulididae } \\
\text { Anchoa sp. } \\
\text { Anchoa argentivittata (Meek \& } \\
\text { Hildebrand) }\end{array}$ & $\begin{array}{l}\text { Lutjanus guttatus (Steindachner) } \\
\text { Lutjanus peru Nichols \& Murphy } \\
\text { Gerreidae }\end{array}$ & $\begin{array}{l}\text { Balistidae } \\
\text { Pseudobalistes polylepis } \\
\quad \text { Steindachner }\end{array}$ \\
\hline Anchoa spinifer (Valenciennes) & Diapterus aureolus (Jordan \& Gilbert) & Tetraodontidae \\
\hline Lycengraulis poeyi (Kner \& & Diapterus peruvianus (Cuvier) & Sphoeroides annulatus (Jordan) \\
\hline Steindachner) & Eucinostomus gracilis (Gill) & Sphoeroides lobatus (Steindachner) \\
\hline
\end{tabular}




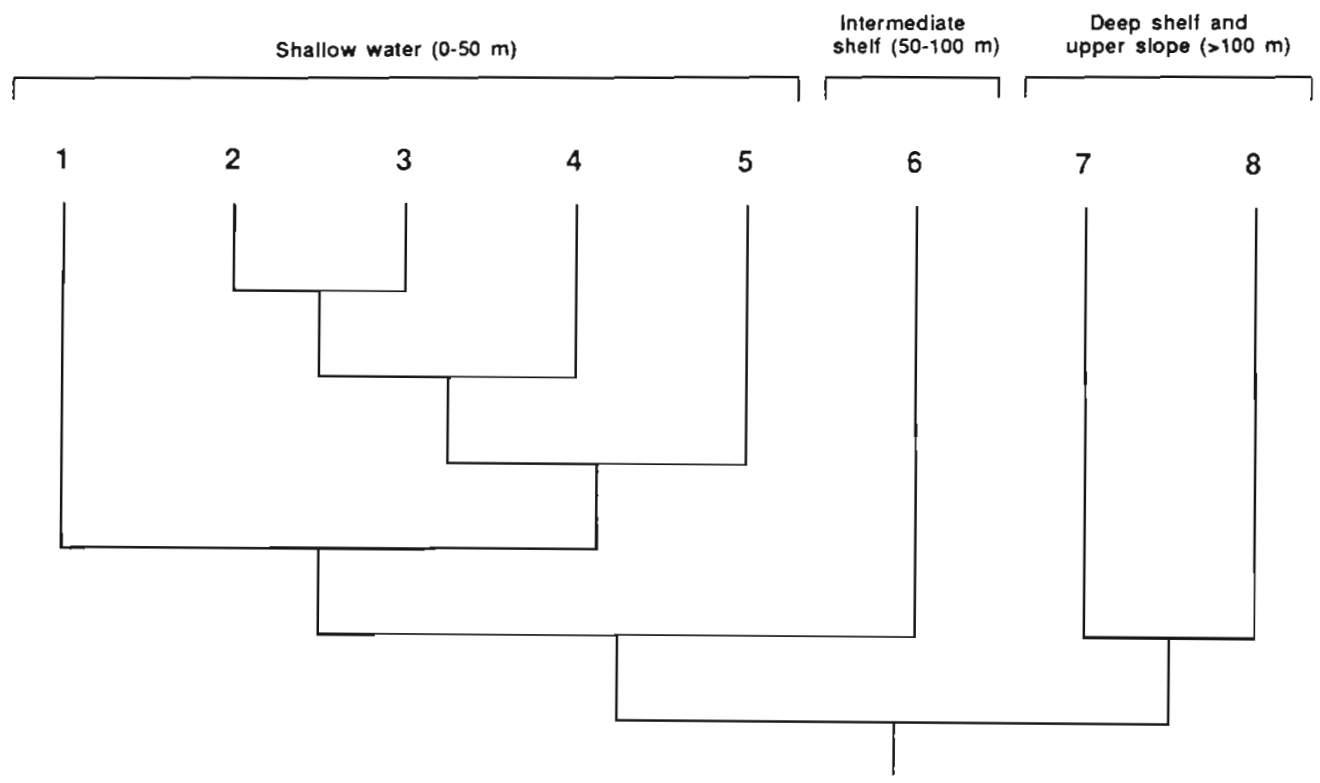

Fig. 4. Dendrogram of station groups (1 to 8) derived from classification with the program TWINSPAN (Hill 1979). See 'Results' for description of each station group

Fig. 6 shows a plot of the station scores on DCA Axis 1 against depth. Although there was a strong correlation between Axis 1 and depth, this correlation was not significant for stations shallower than $50 \mathrm{~m}$ and deeper than $150 \mathrm{~m}$.

In order to improve the resolution of the shallowwater stations, these were further analysed using DCA. Fig. 7 shows the results after extraction of Group 1, which was better separated from the other shallowwater groups. The first 4 eigenvalues were $0.24,0.17$. 0.13 and 0.10 respectively, showing a low degree of separation of these stations, which were indeed very similar in species composition. Results from the correlation of Axes 1 and 2 with depth, temperature, salinity and oxygen are shown in Table 3 . These values clearly

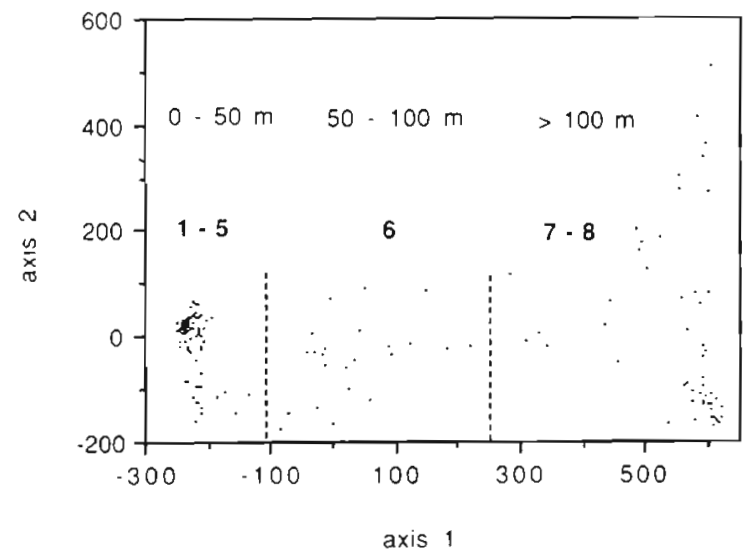

Fig. 5. Detrended correspondence analysis for the AugustSeptember survey (SD units $\times 100$ ). Corresponding TWIA (Two-Way Indicator Species Analysis) groups (1 to 8) and depth ranges also indicated show that faunal changes in shallow-water areas must depend on other factors, such as bottom type, connection to river estuaries, etc.

Comparison of the results from classification analysis (TWIA) to those from ordination analysis (DCA) shows that TWIA Groups 1 to 5 correspond to the first group of

Table 2. Pearson product-moment correlation coefficients between sample scores on DCA (Detrended Correspondence Analysis) Axes 1 and 2 and environmental variables for all stations

\begin{tabular}{lrr|}
\hline Variable & Axis 1 & Axis 2 \\
\hline Depth & 0.96 & -0.10 \\
Temp. & -0.93 & 0.02 \\
Salinity & 0.57 & -0.05 \\
Oxygen & -0.85 & 0.05 \\
\hline
\end{tabular}

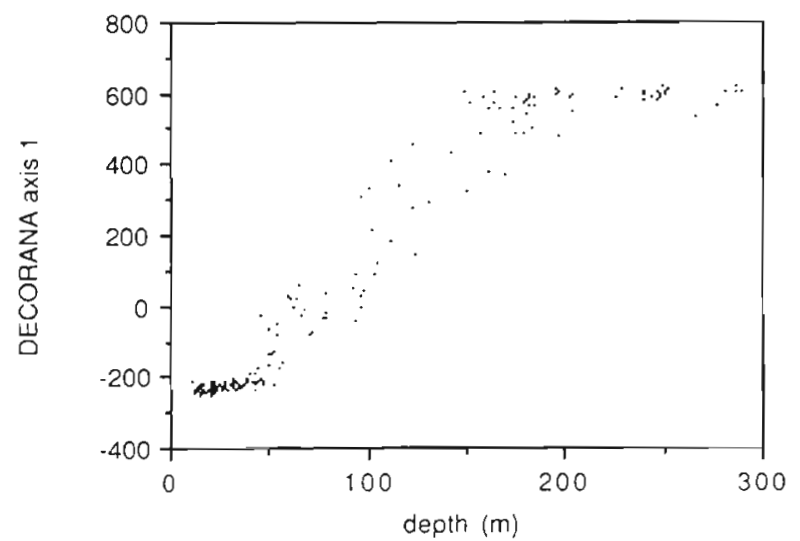

Fig. 6. Plot of station scores on DCA (Detrended Correspondence Analysis) Axis 1 against depth 


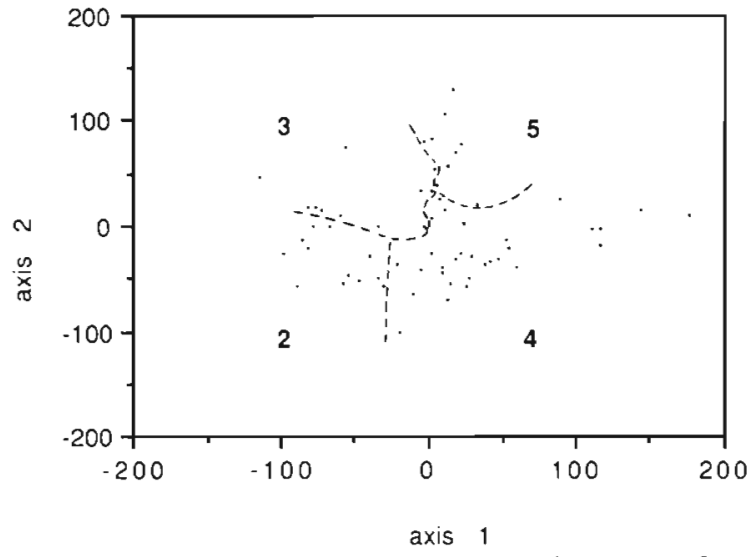

Fig. 7. Detrended correspondence analysis for station Groups 2 to 5 (indicated by numbers) of the August-September survey (SD units $\times 100)$

Table 3. Pearson product-moment correlation coefficients between DCA (Detrended Correspondence Analysis) Axes 1 and 2 and environmental variables for shallow-water stations (Groups 1 to 5)

\begin{tabular}{|lrr|}
\cline { 2 - 3 } Variable & Axis 1 & Axis 2 \\
\hline Depth & 0.13 & 0.70 \\
Temp. & -0.15 & -0.66 \\
Salinity & 0.07 & 0.09 \\
Oxygen & -0.41 & -0.56 \\
\hline
\end{tabular}

DCA, TWIA Groups 6 and (partly) 7 correspond to the second DCA group, and TWIA Group 8 coincides with the third DCA group.

Table 4 presents results from the 'pseudo- $F$ ' test applied to the above groups, together with the average values and standard deviations of the environmental variables. Each station/species group also corresponds to distinct geographical areas, as shown in Fig. 8. Table 5 gives the total weight, numbers and frequency of the main species from each station group.

Fig. 9 shows values of biomass densities obtained with the swept-area method, plotted against depth, for both surveys.

The different groups identified are described as follows:

Group 1: Sandy/shell bottoms off Guatemala and Mexico. This group includes 14 stations and a total of 71 species, located between an area near Salina Cruz (Mexico) and San José (Guatemala), at an average depth of $36 \mathrm{~m}$. This group exhibits a well-defined species composition: it lacks most of the species found in the other shallow-water groups, and is characterized by species whose primary distribution is within this area, including the brassy grunt Orthopristis chalceus, the goatfish Pseudupeneus grandisquamis, the triggerfish Pseudobalistes polylepis, the mojarra Eucinostomus gracilis, the jacks Carangoides ortrynter and Caranx caballus, and the snappers Lutjanus peru and

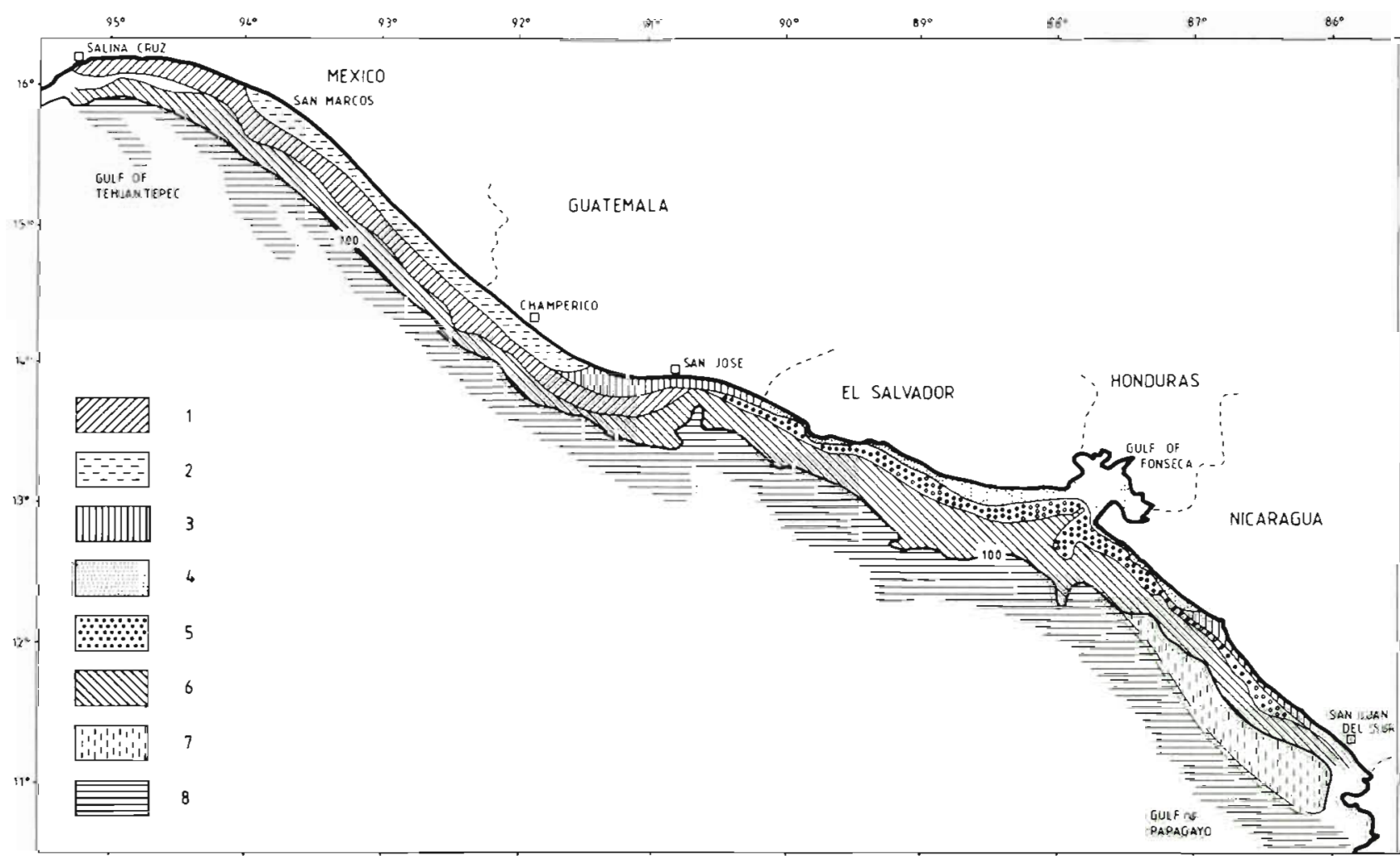

Fig. 8. Subareas corresponding to station groups. Depth gradient in metres 
Table 4. Two-way table based on classification and ordination analyses, showing conforming species groups within site groups. Pseudo-F $(\mathrm{P}-F)$ values preceded by an asterisk indicate conformity at a significance of $\mathrm{p}=0.05$ or better. The average biomass value $(\mathrm{kg})$ of a species within each group, converted to $\ln (x+1)$, is preceded by an asterisk whenever the $95 \%$ confidence interval for the mean is not overlapping. ( $\cdots$ ) Indicates that a species is found only in 1 group. Mean values of environmental variables are also shown for each group, with standard deviations in parentheses. Only the most important species are included

\begin{tabular}{|c|c|c|c|c|c|c|c|c|c|}
\hline \multirow{3}{*}{ Environmental variables } & \multirow[b]{2}{*}{2} & \multirow[b]{2}{*}{3} & \multirow[b]{2}{*}{4} & \multicolumn{2}{|c|}{ Site groups } & \multirow[b]{2}{*}{6} & \multirow[b]{2}{*}{7} & \multirow[b]{2}{*}{8} & \multirow[t]{2}{*}{$P-F$} \\
\hline & & & & 5 & 1 & & & & \\
\hline & & & & & & & & & \\
\hline Depth $(\mathrm{m})$ & $20(5)$ & $25(8)$ & $23(8)$ & $39(6)$ & $37(8)$ & $66(17)$ & $134(33)$ & $205(43)$ & \\
\hline Temp. $\left({ }^{\circ} \mathrm{C}\right)$ & $29(1)$ & $27(2)$ & $28(2)$ & $24(2)$ & $27(2)$ & $22(4)$ & $15(1)$ & $13(1)$ & \\
\hline Salinity (\%) & $33.1(0.3)$ & $33.7(0.5)$ & $33.5(0.5)$ & $33.2(0.5)$ & $33.8(0.4)$ & $34.3(0.3)$ & $34.7(0.2)$ & $34.8(0.0)$ & \\
\hline Oxygen $\left(\mathrm{ml} \mathrm{l}^{-1}\right)$ & $4.5(0.4)$ & $3.6(0.7)$ & $3.6(0.7)$ & $2.5(0.5)$ & $3.1(1.0)$ & $1.8(0.9)$ & $0.7(0.1)$ & $0.4(0.1)$ & \\
\hline Species & & & & & & & & & \\
\hline Anchoa spinifer & & & $\cdot 0.836$ & & & & & & $\cdots$ \\
\hline Bairdiella spp. & 0.191 & 0.228 & $\cdot 1.558$ & 0.561 & & & & & $\cdot 17.5$ \\
\hline Lycengraulis poeyi & & 0.279 & $\cdot 0.948$ & 0.439 & & & & & $\cdot 17.4$ \\
\hline Xiphopenaeus riveti & 0.243 & & $\cdot 1.376$ & 0.203 & & & & & $\cdot 20.2$ \\
\hline Polydactylus opercularis & 1.449 & 1.382 & 0.994 & 1.685 & & 0.022 & & & $\cdot 17.5$ \\
\hline Penaeus vannamei & $\cdot 1.520$ & 0.474 & 0.800 & 0.611 & 0.174 & & & & $\cdot 22.5$ \\
\hline Sphyraena ensis & 2.779 & $\cdot 4.326$ & 2.745 & 2.934 & 0.591 & 0.066 & & & $\cdot 57.5$ \\
\hline Hemicaranx spp. & 0.872 & 1.193 & 1.073 & 0.244 & 0.026 & 0.011 & & & $\cdot 11.3$ \\
\hline Opisthonema libertate & 1.794 & 2.393 & 2.407 & 1.287 & 0.518 & 0.054 & & & $\cdot 26.0$ \\
\hline Opisthopterus dovii & 0.698 & 0.390 & 0.595 & 0.396 & & & & & $\cdot 7.2$ \\
\hline Pliosteostoma lutipinnis & 1.991 & 0.473 & 1.563 & 0.937 & & & & & $\cdot 26.4$ \\
\hline Polydactylus approximans & 1.700 & 2.379 & 1.800 & 1.589 & 0.034 & 0.031 & & & $\cdot 33.8$ \\
\hline Anchoa spp. & 1.827 & 1.457 & $\cdot 2.210$ & 0.331 & & & & & $\cdot 30.1$ \\
\hline Isopisthus altipinnis & 1.006 & 0.671 & 0.964 & 0.754 & & & & & $\cdot 14.2$ \\
\hline Oligoplites refulgens & 0.882 & 0.157 & 0.796 & 0.024 & & & & & $\cdot 14.0$ \\
\hline Anchoa argentivittata & 0.258 & $\cdot 1.349$ & 0.384 & $\cdot 3.280$ & 0.183 & 0.049 & & & $\cdot 22.6$ \\
\hline Larimus acclivis & 1.019 & $\cdot 1.791$ & 0.863 & 0.325 & 0.087 & 0.052 & & & $\cdot 14.4$ \\
\hline Scomberomorus sierra & 1.462 & $\cdot 2.835$ & 1.022 & 1.193 & 0.189 & 0.052 & & & $\cdot 31.7$ \\
\hline Diapterus peruvianus & 3.098 & 3.314 & 1.690 & 1.265 & 0.285 & & & & $\cdot 32.6$ \\
\hline Stellifer spp. & $\cdot 1.207$ & & 0.111 & & & & & & $\cdot 16.3$ \\
\hline Selene peruvianus & 2.083 & $\cdot 3.858$ & 2.010 & 2.734 & 1.014 & 0.211 & & 0.006 & $\cdot 32.6$ \\
\hline Pomadasys panamensis & 0.034 & 1.421 & 0.672 & $\cdot 2.420$ & 0.631 & 0.136 & & & $\cdot 14.3$ \\
\hline Chloroscombrus orqueta & 3.537 & 4.390 & 1.592 & 0.513 & 2.299 & 0.019 & & & $\cdot 57.3$ \\
\hline Pomadasys axillaris & $\cdot 3.044$ & 0.598 & & & 0.495 & & & & $\cdot 50.8$ \\
\hline Diapterus aureolus & 0.342 & $\cdot 1.541$ & 0.168 & 1.326 & 0.224 & 0.167 & & & $\cdot 13.1$ \\
\hline Bagre panamensis & 2.122 & 2.541 & 1.230 & 2.232 & 1.059 & 0.466 & & & $\cdot 20.5$ \\
\hline Carangoides ortrynter & & & 0.006 & 0.199 & $\cdot 1.099$ & & & 0.043 & $\cdot 16.3$ \\
\hline Selar crumenophthalmus & & 0.925 & 0.067 & 0.081 & $\cdot 1.184$ & & & & $\cdot 13.7$ \\
\hline Caranx caballus & 0.361 & 0.631 & 0.267 & 0.114 & 0.763 & 0.030 & & & - 5.0 \\
\hline Eucinostomus gracilis & 0.266 & 0.846 & 0.212 & 0.526 & 1.330 & 0.247 & & & $\cdot 9.0$ \\
\hline Pseudupeneus grandisquamis & 0.283 & 0.497 & 0.176 & 0.454 & $\cdot 1.307$ & 0.106 & & & $\cdot 11.4$ \\
\hline Orthopristis chalceus & 1.136 & 1.505 & & & $\cdot 3.150$ & 0.979 & & & $\cdot 19.3$ \\
\hline Cyclopsetta querna & & 0.563 & 0.166 & $\cdot 2.356$ & 0.013 & 0.469 & & & $\cdot 24.1$ \\
\hline Pseudobalistes polylepis & & & & & $\cdot 1.614$ & 0.244 & & & $\cdot 19.0$ \\
\hline Scorpaena spp. & & & & & & 0.674 & & & $\cdots$ \\
\hline Peprilus snyderi & 1.368 & 2.702 & 2.149 & 3.214 & 0.607 & 1.028 & 2.060 & 0.146 & $\cdot 13.4$ \\
\hline Trichiurus nitens & & 0.079 & 0.535 & 0.071 & & 0.191 & $\cdot 1.160$ & 0.708 & $\cdot 2.9$ \\
\hline Loliolopsis diomedeae & 0.069 & & 0.166 & 0.334 & & 0.806 & 0.383 & 0.033 & $\cdot 3.8$ \\
\hline Porichthys nautopaedium & 0.004 & & 0.083 & 0.077 & & • 1.269 & 0.641 & 0.041 & $\cdot 14.3$ \\
\hline Penaeus brevirostris & & & & & & $\cdot 1.035$ & 0.125 & & $\cdot 15.0$ \\
\hline Lepophidium pardale & & & & & & 0.219 & $\cdot 0.624$ & 0.070 & $\cdot 3.8$ \\
\hline Gobiidae & & & & 0.088 & & 0.029 & $\cdot 1.764$ & 0.020 & $\cdot 24.2$ \\
\hline Citharichthys platophrys & & & 0.034 & & & 0.272 & $\cdot 1.168$ & & $\cdot 14.3$ \\
\hline Prionotus quiescens & & & 0.051 & & & $\cdot 0.862$ & $\cdot 3.426$ & 0.134 & $\cdot 16.3$ \\
\hline Synodus evermanni & 0.139 & & 0.078 & 0.045 & 0.259 & 0.625 & $\cdot 1.248$ & 0.070 & $\cdot 5.8$ \\
\hline Zalieutes elater & & & & & & 0.301 & 0.329 & 0.184 & 1.9 \\
\hline Pontinus sierra & & & & & & 0.009 & 1.844 & 1.548 & $\cdot 14.9$ \\
\hline Squilla biformis & & & 0.056 & & & 0.024 & 0.655 & $\cdot 2.522$ & $\cdot 12.3$ \\
\hline Monolene maculipinna & & & & & & 0.015 & 0.264 & $\cdot 1.513$ & $\cdot 14.7$ \\
\hline Pleuroncodes monodon & & & & & & & $\cdot 1.661$ & $\cdot 5.721$ & $\cdot 63.3$ \\
\hline Merluccius angustimanus & & & & & & & 0.442 & $\cdot 1.628$ & $\cdot 10.9$ \\
\hline Heterocarpus vicarius & & & & & & & & $\cdot 1.120$ & $\cdots$ \\
\hline Argentina aliceae & & & & & & & 0.868 & $\cdot 2.045$ & $\cdot 8.6$ \\
\hline Diplectrum macropoma & & & & & & & & $\cdot 1.420$ & $\cdots$ \\
\hline
\end{tabular}


Table 5. Total weight (W, in $\mathrm{kg}$ ), numbers $(\mathrm{N})$ and frequency ( $\mathrm{F}$ : no. of stations where found in the respective group) of main species from station Groups 1 to 8

\begin{tabular}{|c|c|c|c|c|c|}
\hline Species & W & $(\%)$ & $\mathrm{N}$ & $(\%)$ & F \\
\hline \multicolumn{6}{|l|}{ Group 1 (14 stations) } \\
\hline Chloroscombrus orqueta & 2027 & (35) & 36204 & $(47)$ & 11 \\
\hline Orthopristis chalceus & 897 & $(16)$ & 10550 & (14) & 14 \\
\hline Lutjanus peru & 564 & (10) & 2968 & (4) & 5 \\
\hline Bagre panamensis & 247 & (4) & 2008 & (3) & 6 \\
\hline Pseudobalistes polylepis & 186 & (3). & 1058 & (1) & 10 \\
\hline Eucinostomus gracilis & 171 & (3) & 5590 & (7) & 12 \\
\hline Lutjanus guttatus & 158 & (3) & 204 & (0) & 6 \\
\hline Albula vulpes & 140 & (2) & 372 & (1) & 6 \\
\hline Pseudupeneus grandisquamis & 100 & $(2)$ & 1460 & $(2)$ & 10 \\
\hline Selar crumenophthalmus & 93 & (2) & 804 & (1) & 8 \\
\hline Selene peruvianus & 87 & (2) & 4042 & $(5)$ & 9 \\
\hline Caranx caballus & 47 & (1) & 274 & (0) & 7 \\
\hline Carangoides ortrynter & 39 & (1) & 142 & (0) & 8 \\
\hline Pomadasys panamensis & 18 & (0) & 130 & (0) & 7 \\
\hline Total & 4774 & $(83)$ & 65806 & $(85)$ & \\
\hline Total (all species) & 5764 & & 77674 & & \\
\hline \multicolumn{6}{|l|}{ Group 2 (14 stations) } \\
\hline Chloroscombrus orqueta & 830 & (12) & 14754 & (2) & 14 \\
\hline Selene peruvianus & 680 & (10) & 10732 & (2) & 13 \\
\hline Sphyraena ensis & 629 & (10) & 5410 & (1) & 13 \\
\hline Diapterus peruvianus & 527 & (8) & 7726 & (1) & 14 \\
\hline Pomadasys axillaris & 490 & $(7)$ & 9880 & (1) & 14 \\
\hline Opisthonema libertate & 477 & $(7)$ & 5889 & (1) & 12 \\
\hline Anchoa spp. & 443 & (7) & 402400 & (57) & 11 \\
\hline Orthopristis chalceus & 392 & (6) & 4814 & (1) & 6 \\
\hline Pliosteostoma lutipinnis & 275 & (4) & 198282 & $(28)$ & 11 \\
\hline Peprilus snyderi & 209 & (2) & 1130 & (0) & 7 \\
\hline Bagre panamensis & 177 & (3) & 1892 & (0) & 12 \\
\hline Polydactylus approximans & 167 & (3) & 2604 & (0) & 13 \\
\hline Polydactylus opercularis & 106 & (2) & 866 & (0) & 9 \\
\hline Stellifer spp. & 93 & (1) & 2980 & (0) & 9 \\
\hline Scomberomorus sierra & 76 & (1) & 262 & (0) & 10 \\
\hline Larimus acclivis & 68 & (1) & 1852 & (0) & 7 \\
\hline Penaeus vannamei & 65 & (1) & 1660 & (0) & 14 \\
\hline Isopisthus altipinnis & 57 & (1) & 1028 & (0) & 9 \\
\hline Total & 5761 & $(87)$ & 674161 & (96) & \\
\hline Total (all species) & 6640 & & 704308 & & \\
\hline \multicolumn{6}{|l|}{ Group 3 (11 stations) } \\
\hline Chloroscombrus orqueta & 2442 & $(22)$ & 34372 & $(16)$ & 11 \\
\hline Sphyraena ensis & 1655 & (15) & 11082 & (5) & 11 \\
\hline Selene peruvianus & 1075 & $(10)$ & 26290 & (13) & 11 \\
\hline Diapterus peruvianus & 746 & (7) & 2538 & (1) & 10 \\
\hline Peprilus snyderi & 730 & (7) & 3842 & $(2)$ & 8 \\
\hline Opisthonema libertate & 435 & (4) & 4972 & (2) & 9 \\
\hline Bagre panamensis & 338 & (3) & 1000 & $(0)$ & 10 \\
\hline Scomberomorus sierra & 328 & (3) & 790 & (0) & 10 \\
\hline Anchoa argentivittata & 254 & (2) & 71376 & (34) & 6 \\
\hline Orthopristis chalceus & 251 & (2) & 2890 & (1) & 6 \\
\hline Polydactylus approximans & 228 & (2) & 2280 & (1) & 10 \\
\hline Hemicaranx spp. & 209 & (2) & 1348 & (1) & 5 \\
\hline Pomadasys panamensis & 158 & (1) & 702 & (0) & 5 \\
\hline Diapterus aureolus & 140 & (1) & 4604 & (2) & 8 \\
\hline Anchoa spp. & 97 & (1) & 32333 & (15) & 6 \\
\hline Larimus acclivis & 95 & (1) & 934 & $(0)$ & 9 \\
\hline Polydactylus opercularis & 86 & (1) & 438 & (0) & 6 \\
\hline Eucinostomus gracilis & 25 & $(0)$ & 292 & (0) & 6 \\
\hline Isopisthus altipinnis & 23 & (0) & 182 & (0) & 5 \\
\hline Penaeus vannamei & 13 & (0) & 192 & $0)$ & 4 \\
\hline Pseudupeneus grandisquamis & 11 & (0) & 122 & (0) & 5 \\
\hline Total & 9349 & (83) & 202579 & (96) & \\
\hline Total (all species) & 11205 & & 210926 & & \\
\hline
\end{tabular}


Table 5 (continued)

\begin{tabular}{|c|c|c|c|c|c|}
\hline Species & w & $(\%)$ & $N$ & $(\%)$ & $\mathrm{F}$ \\
\hline \multicolumn{6}{|l|}{ Group 4 (29 stations) } \\
\hline Sphyraena ensis & 903 & $(10)$ & 10101 & (2) & 27 \\
\hline Opisthonema libertate & 879 & $(10)$ & 12557 & (3) & 26 \\
\hline Diapterus peruvianus & 722 & (8) & 2729 & (1) & 18 \\
\hline Selene peruvianus & 577 & (6) & 18159 & (4) & 29 \\
\hline Peprilus snyderi & 543 & (6) & 6388 & (1) & 24 \\
\hline Anchoa spp. & 468 & (5) & 234000 & $(48)$ & 26 \\
\hline Bairdiella spp. & 383 & (4) & 23164 & (5) & 21 \\
\hline Polydactylus approximans & 254 & (3) & 4310 & (1) & 25 \\
\hline Chloroscombrus orqueta & 253 & (3) & 8303 & (2) & 25 \\
\hline Xiphopenaeus riveti & 207 & (2) & 40795 & $(8)$ & 21 \\
\hline Pliosteostoma lutipinnis & 204 & $(2)$ & 53707 & (11) & 26 \\
\hline Larimus acclivis & 204 & (2) & 4382 & (1) & 16 \\
\hline Cynoscion phoxocephalus & 202 & (2) & 2880 & (1) & 11 \\
\hline Hemicaranx spp. & 192 & (2) & 1846 & (0) & 19 \\
\hline Pomadasys panamensis & 138 & (2) & 2575 & (1) & 9 \\
\hline Bagre panamensis & 125 & (1) & 3220 & (1) & 22 \\
\hline Scomberomorus sierra & 125 & (1) & 306 & $(0)$ & 17 \\
\hline Galeichthys peruvianus & 120 & (1) & 453 & (0) & 11 \\
\hline Polydactylus opercularis & 117 & (1) & 1097 & (0) & 18 \\
\hline Parapsettus panamensis & 116 & (1) & 1480 & (0) & 10 \\
\hline Anchoa spinifer & 101 & (1) & 8565 & (2) & 18 \\
\hline Isopisthus altipinnis & 95 & (1) & 4450 & (1) & 20 \\
\hline Oligoplites refulgens & 74 & (1) & 1040 & (0) & 18 \\
\hline Lycengraulis poeyi & 71 & (1) & 1403 & $(0)$ & 25 \\
\hline Penaeus vannamei & 71 & (1) & 3104 & (1) & 18 \\
\hline Trichiurus nitens & 43 & (1) & 2198 & $(0)$ & 15 \\
\hline Total & 7187 & $(80)$ & 452789 & (93) & \\
\hline Total (all species) & 9040 & & 487172 & & \\
\hline \multicolumn{6}{|l|}{ Group 5 (9 stations) } \\
\hline Peprilus snyderi & 838 & (17) & 5943 & (2) & 8 \\
\hline Sphyraena ensis & 722 & (15) & 7567 & (3) & 8 \\
\hline Anchoa argentivittata & 610 & (12) & 217932 & $(76)$ & 8 \\
\hline Selene peruvianus & 354 & (7) & 9321 & (3) & 9 \\
\hline Bagre panamensis & 250 & (5) & 854 & (0) & 6 \\
\hline Pomadasys panamensis & 246 & (5) & 1004 & (0) & 7 \\
\hline Cyclopsetta querna & 158 & (3) & 1025 & (0) & 8 \\
\hline Diapterus peruvianus & 111 & (2) & 320 & (0) & 4 \\
\hline Polydactylus opercularis & 110 & $(2)$ & 698 & (0) & 7 \\
\hline Polydactylus approximans & 90 & $(0)$ & 770 & (0) & 5 \\
\hline Pliosteostoma lutipinnis & 62 & (0) & 18088 & (6) & 4 \\
\hline Opisthonema libertate & 56 & (0) & 1014 & (0) & 7 \\
\hline Diapterus aureolus & 47 & (0) & 2078 & (1) & 6 \\
\hline Scomberomorus sierra & 44 & (0) & 65 & (0) & 5 \\
\hline Isopisthus altipinnis & 19 & $(0)$ & 210 & (0) & 4 \\
\hline Penaeus vamnamei & 10 & (0) & 261 & $(0)$ & 6 \\
\hline Total & 3727 & $(76)$ & 267150 & (93) & \\
\hline Total (all species) & 4935 & & 287095 & & \\
\hline \multicolumn{6}{|l|}{ Group 6 (31 stations) } \\
\hline Loliolopsis diomedeae & 906 & $(22)$ & 204320 & $(65)$ & 12 \\
\hline Orthopristis chalceus & 412 & (10) & 7281 & (2) & 13 \\
\hline Peprilus snyderi & 281 & $(7)$ & 2651 & (1) & 15 \\
\hline Porichthys nautopaedium & 182 & (5) & 16644 & (5) & 26 \\
\hline Penaeus brevirostris & 153 & (4) & 8297 & (3) & 20 \\
\hline Cynoscion reticulatus & 128 & (3) & 135 & (0) & 3 \\
\hline Prionotus quiescens & 122 & (3) & 2218 & (1) & 17 \\
\hline Synodus scituliceps & 112 & (3) & 1826 & (1) & 18 \\
\hline Prionotus horrens & 88 & (2) & 422 & (0) & 6 \\
\hline Synodus evermanni & 86 & $(2)$ & 3122 & (1) & 11 \\
\hline Squilla panamensis & 85 & $(2)$ & 6612 & $(2)$ & 8 \\
\hline Diplectrum labarum & 68 & (2) & 1926 & (1) & 11 \\
\hline Scorpaena spp. & 64 & $(2)$ & 4380 & (1) & 16 \\
\hline Solenocera florea & 39 & (1) & 12708 & (4) & 5 \\
\hline Muraenidae & 21 & (1) & 248 & (0) & 11 \\
\hline Zalieutes elater & 20 & (1) & 1472 & (1) & 11 \\
\hline Total & 2767 & (69) & 274262 & (87) & \\
\hline Total (all species) & 4036 & & 316257 & & \\
\hline
\end{tabular}


Table 5 (continued)

\begin{tabular}{|c|c|c|c|c|c|}
\hline Species & W & $(\%)$ & $\mathrm{N}$ & $(\%)$ & $\mathrm{F}$ \\
\hline \multicolumn{6}{|l|}{ Group 7 (13 stations) } \\
\hline Pleuroncodes monodon & 22583 & $(66)$ & 2935558 & $(90)$ & 5 \\
\hline Prionotus quiescens & 8480 & (25) & 170114 & (5) & 11 \\
\hline Trichiurus nitens & 285 & (1) & 8036 & (0) & 8 \\
\hline Pontinus sierra & 275 & (1) & 9454 & $(0)$ & 9 \\
\hline Gobiidae & 256 & $(1)$ & 27357 & (1) & 8 \\
\hline Peprilus snyderi & 219 & (1) & 6844 & $(0)$ & 11 \\
\hline Synodus evermanni & 161 & (1) & 9050 & $(0)$ & 8 \\
\hline Lepophidium pardale & 71 & $(0)$ & 1952 & $(0)$ & 8 \\
\hline Argentina aliceae & 59 & (0) & 2778 & $(0)$ & 5 \\
\hline Diplectrum euryplectrum & 56 & (0) & 1464 & $(0)$ & 6 \\
\hline Citharichthys platophrys & 56 & $(0)$ & 5792 & $(0)$ & 11 \\
\hline Porichthys nautopaedium & 35 & $(0)$ & 3970 & $(0)$ & 8 \\
\hline Zalieutes elater & 12 & (0) & 1444 & (0) & 8 \\
\hline Monolene maculipinna & 8 & (0) & 1224 & (0) & 5 \\
\hline Penaeus brevirostris & 2 & (0) & 72 & $(0)$ & 5 \\
\hline Total & 32543 & (95) & 3184647 & $(98)$ & \\
\hline Total (all species) & 34209 & & 3258052 & & \\
\hline \multicolumn{6}{|l|}{ Group 8 (55 stations) } \\
\hline Pleuroncodes monodon & 72499 & $(55)$ & 7910418 & $(74)$ & 51 \\
\hline Argentina aliceae & 15406 & (12) & 780158 & $(7)$ & 25 \\
\hline Squilla biformis & 12235 & (9) & 661894 & (6) & 31 \\
\hline Diplectrum macropoma & 11126 & $(8)$ & 610184 & (6) & 25 \\
\hline Heterocarpus vicarius & 2295 & (2) & 249071 & (2) & 18 \\
\hline Merluccius angustimanus & 1339 & (1) & 45308 & $\{0\}$ & 29 \\
\hline Trichiurus nitens & 1101 & (1) & 38273 & $(0)$ & 16 \\
\hline Pontinus sierra & 937 & (1) & 58091 & (1) & 36 \\
\hline Monolene maculipinna & 789 & (1) & 75034 & (1) & 36 \\
\hline Total & 117727 & $(89)$ & 10428431 & $(97)$ & \\
\hline Total (all species) & 132326 & & 10736293 & & \\
\hline
\end{tabular}

L. guttatus (see Appendix 1). The biomass of most of these species is also highest here, as shown in Table 4. The Pacific bumper Chloroscombrus orqueta and the Peruvian moonfish Selene peruvianus, the most widely distributed shallow-water species, are also found here, the former species accounting for $35 \%$ in weight and

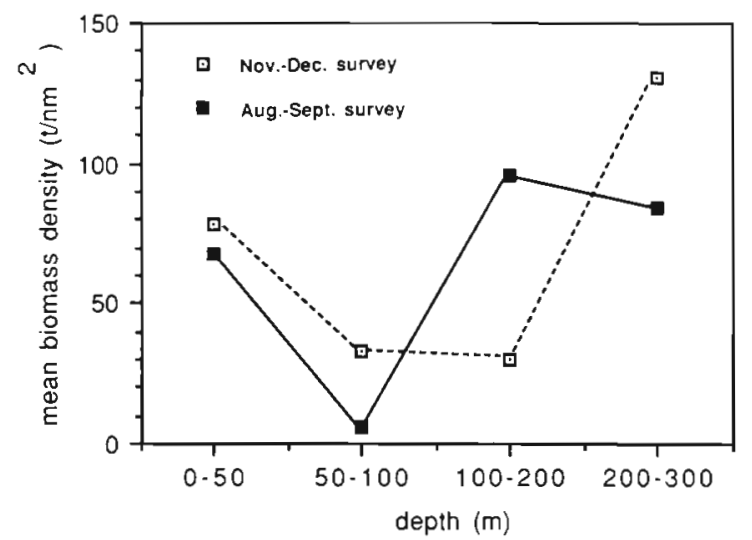

Fig. 9. Plot of mean biomass density (tonnes per square nautical mile) by depth stratum against depth for the AugustSeptember and November-December surveys. Both pelagic and demersal species included for the depth stratum 1-50 m
$47 \%$ in abundance of the total catches for this group (Table 5). Sea charts (Anonymous 1977) report mud/ sand, sandy bottoms with shells and shingle in this area, and some of the above species, for example $L$. peru and $L$. guttatus, are in fact known to prefer hard bottoms (Allen 1985).

Groups 2, 3, 4 and 5 have several species in common: Selene peruvianus, Chloroscombrus orqueta, the threadfins Polydactylus opercularis and $P$. approximans, the whiteleg shrimp Penaeus vannamei, the barracuda Sphyraena ensis, the bluntnose jacks Hemicaranx spp., the thread herring Opisthonema libertate, the weakfish Isopisthus altipinnis, the anchovies Anchoa spp., the catfish Bagre panamensis and the butterfish Peprilus snyder. However, these groups are typified by other species and occupy distinct geographical areas (Fig. 8).

Group 2: Shallow-water stations from near San Marcos (Mexico) to northern Guatemala. This group includes 14 stations, at an average depth of $20 \mathrm{~m}$, with 73 species. A very high bottom temperature $\left(29.2^{\circ} \mathrm{C}\right)$ and high oxygen content (always above $4 \mathrm{ml} \mathrm{l}^{-1}$ ) characterize this area. Typical species are the grunt 
Pomadasys axillaris, Penaeus vannamei and the drums Stellifer spp. The Peruvian mojarra Diapterus peruvianus and Chloroscombrus orqueta are most abundant here and in Group 3 (Table 4). Table 5 shows that the small clupeoids, Anchoa spp. and the yellowfin herring Pliosteostoma lutipinnis, account numerically for about $85 \%$ of the catches in that area. Also, because of their high frequency, they appear as important elements in the food chain, certainly representing an important food item for predators such as Sphyraena ensis and Selene peruvianus. The coastal zone where this group is located is characterized by a series of lagoons which probably serve as nursery grounds for the shrimp (Penaeus vannamei). An important fishery for this species already exists in this area (Holthuis 1980). A more complete list of the species in this group is given in Appendix 1.

Group 3: Shallow waters off Nicaragua and Guatemala. This group is very similar to Group 2, and their separation possibly artificial. Group 3 consists of 11 stations with 77 species. This group is characterized by high concentrations of Sphyraena ensis and the Spanish mackerel Scomberomorus sierra, voracious predators, and Selene peruvianus and Chloroscombrus orqueta, possibly among the prey, with catches often above $1000 \mathrm{~kg} \mathrm{~h}^{-1}$. The mojarras Diapterus aureolus and $D$. peruvianus, Peprilus snyderi, Polydactylus approximans and Bagre panamensis were also almost constantly present in the catches (see Tables $4 \& 5$ and Appendix 1).

Group 4: Gulf of Fonseca and adjacent shallow waters of El Salvador and Nicaragua. This group is well defined and extends from the border between El Salvador and Guatemala to about $12^{\circ} 20^{\prime} \mathrm{N}$ (Nicaragua), including the Gulf of Tehuantepec. It includes 29 stations and 123 species and exhibited a mean depth of $23 \mathrm{~m}$ and high temperature and oxygen levels. Sphyraena ensis, Opisthonema libertate, Selene peruvianus and Diapterus peruvianus are the dominant species, as they are in almost all the other shallowwater Groups 2 to 4 . A number of species are, however, more typical of Group 4: the sabretooth anchovy Lycengraulis poeyi, the croakers Bairdiella spp., Anchoa spinifer and other Anchoa species and the Pacific seabob Xiphopenaeus riveti (Tables $4 \& 5$ ). The soft and muddy bottom as well as the connection to a major river in the southern part of the Gulf explains the presence of the above species. Lycengraulis poeyi is a large anchovy (to $23 \mathrm{~cm}$ total length) and is known to prey on the smaller (to about $7 \mathrm{~cm}$ ) anchovies of the genus Anchoa (Whitehead et al. 1988). The smaller anchovies are the only plankton feeders among the most widespread shallow-water species. These small fishes, because numerous and ubiquitous, must play a significant role in the food chain, and they certainly represent an important food item for the larger predatory species.

Group 5: Deeper shallow waters off Nicaragua and El Salvador. This group, with 9 stations (average depth $39 \mathrm{~m}$ ) and 83 species, can be considered as corresponding to Group 1 (sandy/shell bottoms off Guatemala and Mexico), but is located in the southern part of the area. Most of the widespread shallow-water species missing in Group 1 are present here, particularly Peprilus snyderi, Sphyraena ensis and Selene peruvianus (Appendix 1). Also, the environmental conditions are slightly different, with lower oxygen and temperature levels and a bottom with a much lower sand component. Species most characteristic are Anchoa argentivittata, Pomadasys panamensis and the toothed flounder Cyclopsetta quema (Table 4). This latter species is known to prefer soft bottoms (Chirichigno et al. 1982).

Group 6: Upper intermediate shelf-dwellers. With an average depth of $66 \mathrm{~m}$, much lower oxygen content $\left(1.8 \mathrm{ml} \mathrm{l}^{-1}\right)$ and lower temperatures $\left(21.7^{\circ} \mathrm{C}\right)$, this group appears to be within the range of the thermocline $(35$ to $100 \mathrm{~m}$ ). It includes 31 stations with 127 species. The apparently high number of species is due to the fact that some of the shallow-water species which typify the groups above are also found in the shallower stations of this group, and at the same time, species with a deeper depth range appear in the deepest stations. However, all the above species are present in very small quantities as compared to their respective primary areas of distribution (Table 4). Only 2 species show a clear preference for this area: the small toadfish Porichthys nautopaedium and the crystal shrimp Penaeus brevirostris, with higher biomass than in the other areas. Other species found here were the dart squid Loliolopsis diomedeae, Peprilus snyderi, the searobin Prionotus quiescens, the lizardfishes Synodus scituliceps and $S$. evermanni and the scorpionfishes Scorpaena spp. (Table 5).

Group 7: Lower intermediate shelf-dwellers. This group is found only on the wide shelf off Nicaragua. The 13 stations exhibit average depth, temperature and oxygen values of $114 \mathrm{~m}, 15^{\circ} \mathrm{C}$ and $0.7 \mathrm{ml} \mathrm{l}^{-1}$ respectively. Group 7 contains a total of 54 species; the dominant ones are also found in Group 6 (Peprilus snyderi, Porichthys nautopaedium, Prionotus quiescens, Synodus evermanni, the batfish Zalieutes elater and the sanddab Citharichthys platophrys) or in the deeper shelf and upper slope, such as the scorpionfish Pontinus sierra and the squat lobster Pleuroncodes monodon. This latter species constitutes $66 \%$ by weight and $90 \%$ by number of individuals of the total catch for the stations in this group (Table 5). However, its value in Table 4 is not the highest, because logarithmic transformation reduces the dominant effect of the 2 very large catches which account for the high value of the total catch. 
Group 8: Deeper shelf and upper slope. This group includes 55 stations and 55 species and is characterized by extremely low oxygen levels, ranging from 0.3 to $0.8 \mathrm{ml} \mathrm{l}^{-1}$ (average $0.4 \mathrm{ml} \mathrm{l}^{-1}$ ). Most stations in this group are from the deeper shelf and upper slope of Nicaragua and El Salvador. Pleuroncodes monodon, known in Central and South America as 'langostino', dominates this part of the surveyed area and was caught at extremely high rates (up to $20 \mathrm{t} \mathrm{h}^{-1}$ ) off Nicaragua, while it seemed to be less abundant in the northern part. Biomass of this species, as well as numbers and frequency, was far higher than that of any other species (Table 5). Other species in this group are Pontinus sierra, the deepwater Pacific flounder Monolene maculipinna, the hake Merluccius angustimanus, the argentine Argentina aliceae, the cagua seabass Diplectrum macropoma, the mantis shrimp Squilla biformis and the nylon shrimp Heterocarpus vicarius. Most of these species were not consistently caught at all stations. Argentina aliceae and the cutlassfish Trichiurus nitens were only caught in the daytime hauls.

\section{DISCUSSION}

\section{Species assemblages}

The sharpest changes in species composition occur along the depth gradient, and 3 major zones of the continental shelf can be identified. The upper zone (to about $50 \mathrm{~m}$ depth), with oxygen values usually well above $2 \mathrm{ml} \mathrm{l}^{-1}$, is rich in number of species (well over 200 ) and exhibits relatively high biomass densities. The intermediate zone (to about $100 \mathrm{~m}$ ), widely influenced by the thermocline and thus displaying rapid changes and short-term fluctuations in physical characteristics of the water masses, still contains a high number of species (about 160), but most of them have their optima in the water layers above and below this level, and biomass densities are in fact very low here. The deeper zone has an extremely low oxygen content (usually $<1 \mathrm{ml} \mathrm{l}^{-1}$ ), which is probably the main factor, together with bottom type, explaining the type of fauna found. A single species, Pleuroncodes monodon, dominates the environment, together with Squilla biformis and Heterocarpus vicarius, present in much smaller quantities. It seems that the above crustaceans, particularly P. monodon, are well suited to live in hypoxic conditions. Of the few fish species found here in considerable quantities - Trichiurus nitens, Argentina aliceae and Diplectrum macropoma - the first 2 are known to perform daily vertical migrations.

Analysis of the November-December survey broadly confirms the results obtained from the August-Sep- tember survey. However, faunal discontinuities along the depth gradient are less clear. The largest gap on DCA Axis 1 separates the stations deeper than $150 \mathrm{~m}$ from the others. This seems to be due mainly to the migration of Pleuroncodes monodon to slightly greater depths.

Further separation of groups, within each depth stratum, is less marked. Of the shallow-water stations, Groups 1 to 5, only Group 1 (found on sandy/shell bottoms of southern Mexica) is quite distinct. The remaining groups display a very similar species composition, although with significant differences in those species' relative abundances (Table 4). Group 4 (Gulf of Fonseca and adjacent coastal areas) is also distinguished by a number of species (Lycengraulis poeyi, Anchoa spinifer, Xiphopenaeus riveti and Cynoscion phoxocephalus) highly characteristic for this area.

Most of the studies on demersal fish assemblages on continental shelves have indicated that the main faunal changes occur along the depth gradient (Fager \& Longhurst 1968, Lleonart \& Roel 1984, McManus 1985, Roel 1987). Physical characteristics of water masses, as well as bottom type, light intensity, pressure, etc., are mostly depth-dependent, and depth obviously reflects the combined effects of these factors. Fager \& Longhurst (1968) found that separation between different assemblages in the Gulf of Guinea was related to the thermal discontinuity layer as well as to sediment type (which also changed with depth). McManus (1985) studied fish assemblages of the Samar Sea (Philippines) from 20 to $90 \mathrm{~m}$ depth and found a depth-dependent faunal distinction between 30 and $40 \mathrm{~m}$, independent of season and substrate type. Lleonart \& Roel (1984) identified structures in species composition associated with depth and latitude when analysing demersal communities of fishes and crustaceans of the Namibian coast. Roel (1987) also concluded that composition of the demersal fauna in the upwelling region off South West Africa was related mainly to depth. In this respect, he found a main boundary between the slope fauna and the shelf fauna at about $380 \mathrm{~m}$ depth. The area corresponding to the shelf community could be further subdivided into 5 subareas. Two of these extended over the whole shelf and did not seem to be subject to seasonal variations. The remaining 3 corresponded to the inner shelf; their extent varied between summer and winter and appeared to be independent of depth.

The above studies, as well as the present one, indicate that when the depth range is wide enough to include areas where different water layers impinge on the shelf slope, the greatest changes in species composition are depth-related. However, within each water layer, other factors - such as presence of river mouths, type of substratum, etc. - become more relevant. 


\section{Biomass}

The highest biomass densities are found along the continental shelf-slope boundary. Longhurst \& Pauly (1987) indicate that the distribution of benthic biomass on tropical continental shelves reflects the importance of inshore primary production and/or enrichment from rivers, and that highest benthic biomass values correspond to the inshore mixed layer. Here, regeneration of nutrients from the bottom can be directly utilized for phytoplankton production. This is the case, for example, in the tropical Atlantic (Guinea-Sierra Leone), where Longhurst (1959) found highest benthic biomass in shallow inshore waters and a minimum at the bottom of the thermocline (50 to $100 \mathrm{~m}$ ). Rowe (1971) found an inverse relationship between biomass and depth in temperate regions, such as the north temperate Atlantic, in tropical regions such as the Gulf of Mexico, and in the upwelling area of the Pacific off Peru. However, the conclusions apply to a wide depth range (shelf to over $5000 \mathrm{~m}$ depth), and a high variance was found in waters shallower than $1000 \mathrm{~m}$ in the upwelling area off Pisco (Peru). Here the influx of organic material is so high as to cause oxygen depletion, and stressful conditions for life and maximum biomass densities are found offshore of the oxygen-poor depths. The conditions found in the area under study seem to represent another case of deviation from the general trend of biomass decreasing with depth. As shown in Fig. 9, highest biomass densities are found below $100 \mathrm{~m}$ depth, consisting mainly $(80 \%)$ of Pleuroncodes monodon. The survey report (Strømme \& Sxtersdal 1988) gives identical catch rates for both daytime and nighttime hauls, which suggests a strictly demersal behaviour for this species.

The reason for this apparent deviation can be deduced from the fact that the region under study, although geographically tropical, is characterized by singular hydrographic conditions. High productivity results from the processes described above (seasonal upwelling and shallow thermocline; see 'Study area') and, possibly under-utilized by pelagic herbivores, is deposited by sedimentation on the bottom. Haedrich et al. (1976) as cited by Rowe (1981) also reported highest densities of large benthic organisms (megafauna) along the continental shelf/slope boundary off northern West Africa, and they related this finding to a prominent shelf-break upwelling. It seems evident that the inverse relationship between depth and benthic biomass is to be considered a general trend but that local hydrographic conditions may introduce deviations from this pattern.

Mass occurrences of anomouran crustaceans have been reported from other regions, usually highly eutrophic, like the California Current and the Hum- boldt Current. The dominance of this group seems to be due mainly to their wide range of feeding mechanisms and their ability to live in oxygen-deficient waters. Benthic mass aggregations tend to occur in areas below diatom blooms and at depths corresponding to those where the oxygen-minimum layer meets the continental shelf/slope (Longhurst 1968). Low oxygen content may be responsible for a lower number of taxa and, because of reduced competition, for high densities. Pleuroncodes monodon, in particular, is also very abundant off central Chile, where it occurs on the deeper shelf together with another galatheid (Cervimunida johni) and the shrimp Heterocarpus reedi (Longhurst 1968). Here the environmental conditions are similar to those found in the area of the present study - i.e., oxygen-deficient waters and high productivity. However, these are not so pronounced as to produce practically anoxic conditions with a rich fauna of sulphur bacteria, such as those further north off northern Chile and Peru, where 'semiabiotic' regions can be found between the deeper shelf and upper slope areas (about 100 and $500 \mathrm{~m}$ depth) (Rowe 1971).

\section{Taxonomic note}

The Pleuroncodes species found in this area has usually been identified as $P$. planipes Stimpson (Vidal 1971. Orellana \& Escoto 1981 and others). This species typifies the Baja California upwelling region, while $P$. monodon ( $\mathrm{H}$. Milne Edwards) has been considered to be the southern-hemisphere congener. Highest concentrations of this species are found off central Chile, at depths between 125 and $200 \mathrm{~m}$ (Longhurst 1968). However, Boyd (1963), as cited by Longhurst (1968), had already reported the occurrence of $P$. monodon off the west coast of Mexico and hypothesized that this species possibly occurs off Central America. This was later confirmed by Longhurst \& Seibert (1971), who found young stages of $P$. monodon in micronekton nets during eastern tropical Pacific expeditions.

Pleuroncodes planipes are known to occasionally form large pelagic swarms as adults, while most species belonging to the same family (Galatheidae) are exclusively benthic. In the course of the RV 'Dr. F. Nansen' surveys Pleuroncodes $\mathrm{sp}$. Was caught only in the bottom trawl. Also, none of the pelagic acoustic recordings was attributed to this species. No significant differences were found in the catches between night and day, confirming the strictly demersal nature of the population found off Central America

Specimens collected off Nicaragua in the course of the RV 'Dr. F. Nansen' survey programme were recently analysed by $M$. Türkay (Senckenberg Museum, Frankfurt, Germany), who found (pers. comm.) 
that the species in fact fits the description of Pleuroncodes monodon, i.e. displays slightly flattened and bare pereiopods, compared to the extremely flattened and ciliated pereiopods of $P$. planipes.

I believe that the population off central America is in fact Pleuroncodes monodon and that the appellation. 'planipes' has been erroneously used in this area.

Acknowledgements. I thank T. Høisæeter for his help in improving early versions of this manuscript with critical comments and helpful suggestions; T Strømme for developing some of the programs utilized in the course of this study; J. H. B. Birks for guidance in choosing the most appropriate multivariate analysis techniques and for providing a more powerful version of the program TWINSPAN $N_{i}$. Türkay for kindly analysing the Pleuroncodes specimens deposited at the Senckenberg Museum (Frankfurt); G. Sætersdal, R. Mahon, S. Garcia, G. Sharp and K. Dorsey, who have read the manuscript and provided further useful comments; and S. Kolbeinson, Institute of Fisheries Biology, University of Bergen, for drawing the maps.

\section{LITERATURE CITED}

Allen, G. R. (1985). FAO species catalogue, Vol. 6, Snappers of the world. An annotated and illustrated catalogue of lutjanid species known to date. FAO Fish. Synopses $125(6)$ : $1-208$

Anonymous (1977). Admiralty charts. Central America West Coast, N. 587. Hydrography of the Navy, Somerset, UK

Bartels, C. E., Bussing, W. A., Lopez, M. I., Price, K. S. (1983) Occurrence, distribution abundance and diversity of fishes in the Gulf of Nicoya (Costa Rica). Revta Biol. trop. 31: 75-101

Blackburn, M. (1966). Biological oceanography of the Eastern Tropical Pacific: summary of existing information. Spec. scient. Rep. US Fish. Wildl, Serv. (Fish.) 540: 1-18

Boyd, C. M. (1963). Distribution, trophic relationships, growth and respiration of a marine decapod crustacean, Pleuroncodes planipes Stimpson (Galatheidae). M.Sc. Thesis, University of California, San Diego

Brandhorst, W. (1958). Thermocline topography, zooplankton standing crop, and mechanisms of fertilization in the eastern tropical Pacific. J. Cons. perm. int. Explor Mer 24: $16-31$

Caddy, J. F., Sharp, G. D. (1986). An ecological framework for marine fishery investigations. FAO Fish. tech. Pap. 283 $1-152$

Chan, E. H., Liew, H. C. (1986). Characteristics of an exploited tropical shallow-water demersal fish community in Malaysia. In: MacLean, J. L., Dizon, L. B., Hosillos, L. V. (eds.) The first Asian Fisheries Forum. Proceedings of the first Asian fisheries forum, Manila, Philippines, 26-31 May, 1986. Asian Fisheries Society, Manila, p. 349-352

Chavez, E. A. (1979). Análisis de la comunidad de una laguna costera de la costa suroccidental de México. An. Cent. Cienc. Mar Limnol., Univ nac. Autónoma México 6 (2): $15-44$

Chirichigno, N., Fisher, W., Nauen, C. E. (1982). INFOPESCA. Catálogo de especies marinas de interés económico actual o potencial para América Latina. Parte 2 - Pacífico centro y suroriental. FAO/PNUD (Programa dé las Naciones Unidas para el Desarrollo), SIC/82/2 (Servicio de Información para la comercialización de los productos pesqueros en América Latina), Rome

Fager, E. W., Longhurst, A. R. (1968). Recurrent group analysis of species assemblages of demersal fish in the Gulf of Guinea. J. Fish. Res. Bd Can. 25: 1405-1421

Glynn, P. W., Druffel, E. M., Dumbar, R. B. (1983). A dead central American coral reef tract: possible link with the Little Ice Age. J. mar Res. 41. 605-637

Green, R. H., Vascotto, G. L. (1978). A method for the analysis of environmental factors controlling patterns of species composition in aquatic communities. Wat. Res. 12: 583-590

Jongman, R. H. G., ter Braak, C. J. F., van Tongeren, O. F. R. (1987). Data analysis in community and landscape ecology. Pudoc Wageningen, The Netherlands

Haedrich, R. L., Blackburn, M., Bruhlet, J. (1976). Distribution and biomass of trawl-caught animals off Spanish Sahara, West Africa. Matsya (Bull. Indian Soc. Ichthyologists) 2 : $38-46$

Hill, M. O. (1979) TWINSPAN - A FORTRAN program for arranging multivariate data in an ordered two-way table by classification of individuals and attributes. Cornell University, Ithaca, New York

Hill, M. O., Gauch, H. G. (1980). Detrended correspondence analysis, an improved ordination technique. Vegetatio 42 : $47-58$

Holthuis, L. B. (1980). FAO species catalogue, Vol. 1, Shrimps and prawns of the world. An annotated catalogue of species of interest to fisheries. FAO Fish. Synopses 125 (1): $1-261$

Lleonart, J., Roel, B. A. (1984). Análisis de las comunidades de peces y crustáceos demersales de la costa de Namibia (Atlántico Sudoriental). Investigación pesq. 48: 187-206

Longhurst, A. R. (1959). Benthos densities off tropical West. Africa. J. Cons. int. Explor Mer 25: 21-28

Longhurst, A. R. (1968). The biology of mass occurrences of galatheid crustaceans and their utilization as a fisheries resource. FAO Fish. Rep. 57: 95-110

Longhurst, A. R., Pauly, D. (1987). Ecology of tropical oceans. Academic Press, New York

Longhurst, A. R., Seibert, D. L. R. (1971). Breeding in an oceanic population of Pleuroncodes planipes (Crustacea, Galatheidae). Pacif. Sci. 25: 426-428

McManus, J. W. (1985). Descriptive community dynamics background and an application to tropical fisheries management. Ph.D. thesis, University of Rhode Island, Providence

Orellana, F. T., Escoto, R. G. (1981). Crucero de evaluación del recurso langostino Pleuroncodes planipes. Boletín Técnico Instituto Nicaraguense de la Pesca. Dirección de planificación, Investigación y Desarrollo, Managua

Rainer, S. F. (1984). Temporal changes in a demersal fish and cephalopod community of an unexploited coastal area in northern Australia. Aust. J. mar. Freshwat. Res. 35: $747-768$

Rainer, S. F., Munro, I. S. R. (1982). Demersal fish and cephalopod communities of an unexploited coastal environment in northern Australia. Aust. J. mar. Freshwat. Res. 33: 1039-1055

Roden, G. I. (1961). On the wind driven circulation in the Gulf of Tehuantepec and its effect upon surface temperatures. Geofísica Internacional, México 1-55-76

Roel, B. A. (1987). Demersal communities off the west coast of South Africa. In: Payne, A. I. L., Gulland, J. A., Brink, K. H. (eds.) The Benguela and comparable ecosystems. S. Afr. J. mar. Sci. 5: 575-584

Roel, B. A., Rucabado, J., Lloris, D., Lleonart, J. (1985). Las comunidades de peces demersales del afloramiento de 
Appendix 1. Two-way station-by-species table produced by the program TWINSPAN (Hill 1979). Values denote categories of abundance (in weight, W): (1) $W<10 \mathrm{~kg}_{i}$ (2) $10<W \leqslant 100 \mathrm{~kg}$; (3) $100<W$ $\leqslant 1000 \quad \mathrm{~kg}_{i} \quad$ (4) $1000<W$ $\leqslant 10000 \mathrm{~kg}_{i}(5) \mathrm{W}>10000 \mathrm{~kg}$. Vertical lines separate station groups; station numbers along top margin (arranged vertically under group numbers). Hierarchy shown in binary notation along the bottom and right margins of the table for stations and species respectively
2

3

$1111111111111 \mid 111111111111111_{11} 1111$

Cynoscion proxocephalus opisthopterus spp. Lycengraulis poey. Bairdiella spp. Anchod spinifer Xiphopenaeus riveti Parapsetcus panamensis
Polydactylus opercularis Penaeus vannamei

Sphyraena ensis

Hemicaranx spp.

Hemicaranx spp.

Opisthonema libertace

Opisthopterus dovit Polydactylus approximans Isopisthus altiplnnis

opisthopterus equitor

Anchoa spp.

Oligoplites refulgen

Pomadasys leuciscus

Neoopisthopterus tropicus

Anchos argentivis acclivis

Scamberamorus sierro

Larimus gulosus

Lariaus gulosus

Xenichenys xanti

conodon macrops

stellifer spp.

Lartmus effulgens

Larjaus effulgens
Luejanus gutcatus

Albula vulpes

Selar crumenophthalmus

Caranx caballus

selene peruvianus

pomadasys panamensis

Caranx caninus

Caranx caninus
Chioroscambrus orqueta
Chaetodipterus zonacus

sphyrna lewini

Pomadasys axillaris

pomadasys axillaris

cynoscion reticulatus

Micropogonias altipinnis

Bagre panamensis

Arius spp.

Carcharhinus porosus

Lalliguncula panamensis

Carangoldes ortrynter

Eurinostamus gracilis

Galeichthys peruvianus

Pseudupeneus grandisquan

orthopristis chalceus

cyclopseted querna

Luejanus peru

Nasolanta velax

Sphoeroldes annulatus

Portunus acuminatus

Pseudobalistes polylepis

Scorpaena spp.

Prionotus horrens

penaeus callforniensis

Sphoeroides lobatus

Synodus scituliceps

Peprilus snyderi

Portunus asper

Mraenidae

Diplectrum labarum

Lollolops is diomedeae

Porlchehys nautopaedium

Penaeus brevirostris

Ophichthidae

squillo paramensis

Squille panarensis
symphurus atramentatus

Lepophidium pordale

Lepophidiur

Citharichthys platophrys

prionotus quíscens

synodus evermanni

Synodus evermann

Symphurus spp.
Symphurus elongatus

Symphurus elongatus
Diplectruat euryplectrum

Dlplectruat euryp
zalleutes elater

Lophiodes caulinaris

Solenocera agassizí

Solenocer agassiz
pronotogranmus eos

Pronotogramaus eos

Mursia gaudichaudí

Morldae

Morldae

olplectrum macropomo

Opplectrum macropoma

Hemanthias signifer
Monolene maculipinna

Monolene maculipinna

Merlucclus anqustimonus

Merlucclus

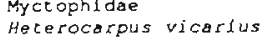

Heteracarpus vica
Argentina aliceae

Argentina aliceas
squilla biformis

trichlurus nitens

\section{-}

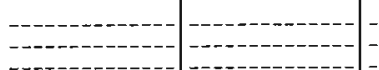

-

1.-

$-212---111-11$

$-1---1211-12$

$-1--11111-2-22$

$-111--1---11-1$

$-214212-1121$

$--2--1-\sim-2-1$

$-1---3-1--$

$------1--$

$111-12-1-21-1$

$11-12-1-21-1$$$
2
$$

$-21122-\cdots-1$ $---1----1---$

$-1---11-1112-1$

$-1211113-2111$

$-21--1-11-$

s-1222111111--

-

$22-1-2-\cdots---$

$---1-\cdots----$

$-1-21-1-112222$

$--1-------$

$--1-11--1--$

$---1------1$

$-0-1-0$

--

-

-

-

-...-

-

-

-

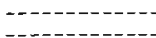

-...-

-

-

-

-

-

-

-

$111---1-0$

-

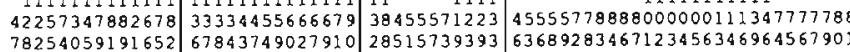

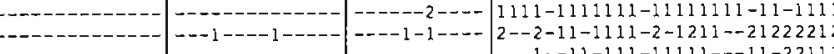

$-11---1-2--1-(--1-11-111-11111---11-2211$

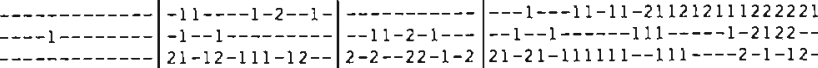

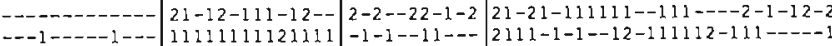

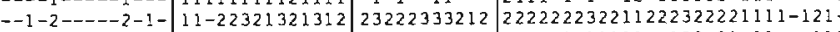

--111112--11- -2-132--1-- 1--121-11111--122-21-11---11

-2-2----- $111331-111111 \mid 23-1222121-2112311221221231211-2211-121$

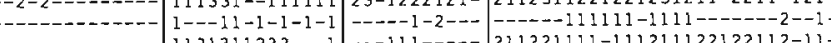

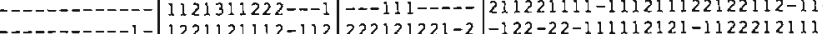

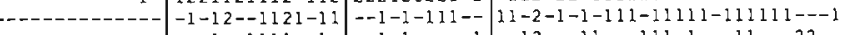

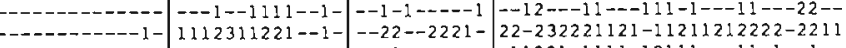

\begin{tabular}{l|l|l|l|l}
- & 1 & 1 &
\end{tabular}

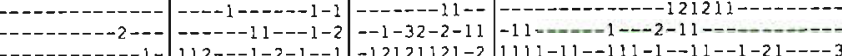

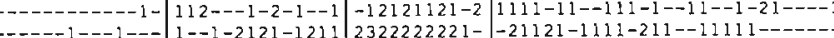

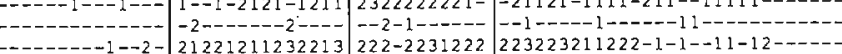

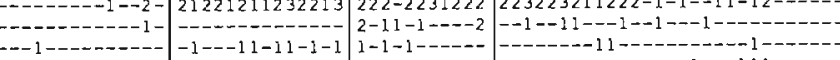

-

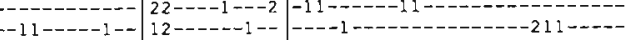

$213312111111-1 \quad 233222221301222113111111111111213112112$

$-23----2111$

0000000000000 0000000000000 0000000000000

00000000111 00011111

\section{$22322221221322 \quad 33222332234$}

$1121111111212211--1112112-1$

$-2=----2-$

\begin{tabular}{l|l}
$-2111-121-2$ \\
$-2111-31-2$
\end{tabular}

$11----1-1-1-$

$-11--1---1$

$11---1$

$-1-2---1---1$

231221

-

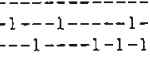

-

$1-2 .-11-$

$21-1-2312--$

$112-2312--$

$-0-1-1-$

$-11--0-0-1$

-

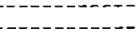

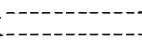

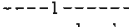

$1+2-1--1-1-$

-

(n-

(n)

-

-

-

$-122---1$

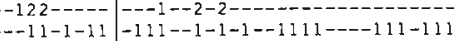

$-1--11121$

$1-1--111-$

$2211-\cdots-2-3$

$-1-12--11-1-----1-1-----2$

$---2-21--$

$1-1---1--1-$

-1.n-

-

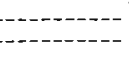

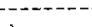

$331-2-321-$

-

$--1---1--$

$-2-2-223111212222221112-221$

$--1$

$-1-1-0-1$

$--1-0-1$

-

-

-

-

-

-

-

-

(-)-

$-1--1--$

000000000000000000000000000000000000000000000000000000 000000000000000000000000000000000000000000000000000000 \begin{tabular}{l|l|l|l|}
1111111111111 & 11111111111 & 111111111111111111111111111 \\
00000000000000 & 00000000000 & 11111111111111111111111
\end{tabular}

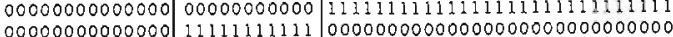
$0001111111111100111111111 \mid 00000000000000000000001111111$

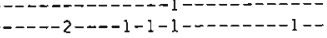


\begin{tabular}{r|ccc}
111 & 11111111111111 & 11 \\
4 & 1
\end{tabular} 134452278850328836751347458801019018720

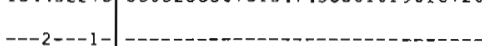

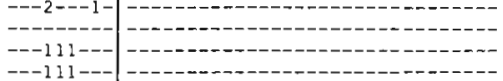

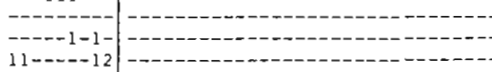

$222-2111-$

$122233-21$
$-1-1---1$

$-1-1---1$
-1112111

$-11-1-1$

$-212-1$

$-22212-$

$-111-1-$

-

13332312

$13332312-$

$21-1---11$

$---2-121$

-

-

32222121

32222121

23212

-1---2---

$----1-21$
$-2-1-111-1$

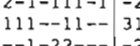

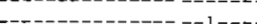

---222211 -1211--1---1--1------1-1--1--

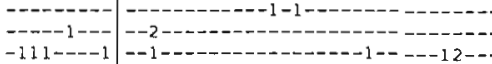

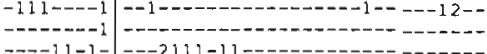

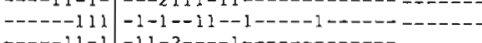

$-1-12321-222-1--12---1-----$

$21212221-211----+--1--111-1--11--1-0$

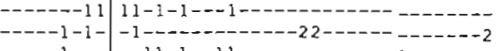

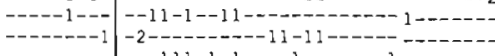

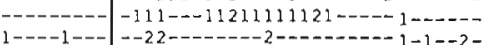

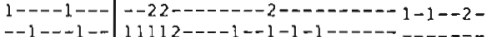

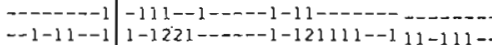

$3222321-1 \mid-111-----12211221121-2$

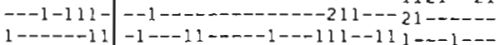

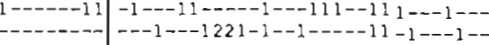

$--1-111-0-1--122-1--1-11121-1--233$

$-22211--1221-1111111-11222111-2$

- $1-1--12-1--2-11112111221211--$

$-0-1--1----1-11-112--221-1--1$

-

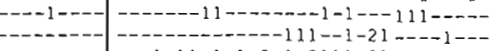

$---1-1-11-1-1-2-1-2111-21211-1--$

$-1-0 .-1-1$

-

-

-2-1-20

-

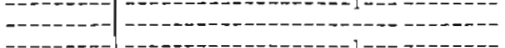

\begin{tabular}{l|l}
- \\
-
\end{tabular}

-

-

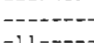

0000000000000000000000000000000000000000 000000000111111111111111111111111111111 1111111120000000000000000011111111111111 1111111110000011111111111100000000000111 1111111110011100000001111100000011111 000000111000111110001100011100011 \begin{tabular}{ll|lll}
11 & 111111 & 111111 & 11 & 11
\end{tabular} 89921874131673520285463369956989645028050126516096570247015748243492

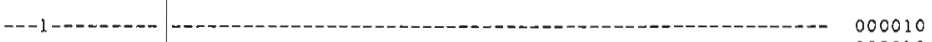

-

-

-

-

-

-2.00
-

000100

000100

000100

000100

000100

000100

000100

000100

000100

000100
000100

000101

000101

000101

000101

000101

000101

000110

000110

001000

001000
001000

001000

001000

001000

001001

001001

001001

001001

001001

001001
001010

001010
001010

001010

001010

001011

0011

0011

010001

010001

010100

010100

010101

010101

010101

010101

010101

010110

010110

010110

010110
010111

011

011

100000

100001

100001

100001

100001

100010

10001

1001

1010

10110
10110

10110

1011 ?

11000

11010

11010
11011

111000

111000

111001

111001
111010

111010
111010

111010

111011

111011
111011

111011

111011

111011
111011

111011

11101

$--1---12-22$

1-1-1---11223

1111111111111

\begin{tabular}{l|l}
0000000000000 & 1111111111111111111111111111111111111111111111111 \\
0000000011111 & 000000000000000000000000000000000000000000011111111
\end{tabular}

00000011001110000000000000000000000000000000011111111111111000000001

000000011111111111111111111100000000000000100000011

0 o 11111000000000000000111111110000011111111000111 
África occidental (Sahara y Namibia). In: Bas, C., Margalef, R., Rubies, P. (eds.) 1985. Simp. Int. Afl. O Afr., Vol 2. Inst. Inv. Pesq., Barcelona 2: 691-699

Rowe, G. T (1971). Benthic biomass and surface productivity In: Costlow, J. D. (ed.) Fertility of the sea. Vol. II. Gordon and Breach Science Publishers, New York, p. 441-454

Rowe, G. T (1981). The benthic processes of coastal upwelling ecosystems. In: Richards, F. A. (ed.) Coastal upwelling Coastal and estuarine sciences, Vol. 1. American Geophysical Union, Washington, D.C., p. 464-471

Sainsbury, K. J. (1987). Assessment and management of the demersal fishery on the continental shelf of northwestern Australia. In: Polovina, J. J., Ralston, S. (eds.) Tropical snappers and groupers. Biology and fisheries management. Ocean Resources and Marine Policy Series, Westview Press, Boulder, Colorado, p. 465-503

Sameoto, D. D. (1986). Influence of the biological and physical environment on the vertical distribution of mesozooplankton and micronekton in the eastern tropical Pacific. Mar Biol. 93: 263-279

Stromme, T., Sætersdal, G. (1988). Surveys of the fish resources on the Pacific shelf from Colombia to Southern Mexico 1987. Reports on surveys with RV 'Dr. F. Nansen' Vol. 1. 96 pp.; Vol. 2, Data File. Institute of Marine Research, Bergen, Norway

Subba Rao, D. V., Sameoto, D. D. (1988). Relationships between phytoplankton and copepods in the deep tropical Pacific Ocean off Costa Rica. Bull. mar. Sci. 42: 85-100

ter Braak, C. J. F. (1987). CANOCO - a FORTRAN program for canonical community ordination by (partial) (detrended) (canonical) correspondence analysis, principal

This article was submitted to the editor components analysis and redundancy analysis (version 2.1). ITI-TNO, Wageningen, The Netherlands

ter Braak, C. J. F., Prentice, J. C. (1988). A theory of gradient analysis. Advances ecol. Res. 18: 271-317

Vidal, J. J. (1971). Algunas características biológicas de camarones y langostino de profundidad en el Pacífico de Centro América. Proyecto Regional de Desarrollo Pesquero en Centro América, Circular 71/12. CCDP-FAO-PNUD, San Salvador

Warburton, K. (1978). Community structure, abundance and diversity of fish in a coastal lagoon system. Estuar. coast. mar. Sci. 7: 497-519

Whitehead, P. J. P., Nelson, G. J., Wongratana, T (1988). FAO species catalogue, Vol. 7. Clupeoid fishes of the world (Suborder Clupeoidei). An annotated and illustrated catalogue of the herrings, sardines, pilchards, sprats, anchovies and wolfherrings. Part 2, Engraulididae. FAO Fish. Synopses 125 (7, Part 2): 305-579

Wyrtki, K. (1967). Circulation and water masses in the Eastern Equatorial Pacific Ocean. Int. J. Oceanol. Limnol. 1: $117-147$

Yanez-Arancibia, A. (1978a). Ecological patterns and cyclical variations of the trophic structure in nektonic communities in coastal lagoons of the Pacific coast of Mexico. An. Cent. Cienc. Mar Limnol., Univ. nac. Autónoma México 5: 287-306

Yanez-Arancibia, A. (1978b). Taxonomía, ecología y estructura de las comunidades de peces en lagunas costeras con bocas efímeras del Pacífico de México. An. Cent. Cienc. Mar Limnol., Univ nac. Autónoma México (Spec. Publ. No. 2), p. 1-300

Manuscript first received: December 4, 1990 Revised version accepted: April 11, 1991 